\title{
Nonlinear Analysis in Synthesis Theory of Radiating Systems
}

\section{Petro Savenko}

Department of numerical methods of the Pidstryhach Institute for Applied Problems of Mechanics and Mathematics, NASU,3 «B» Naukova Str., 79060, Lviv, Ukraine E -mail: spo@iapmm.lviv.ua

\begin{abstract}
A brief overview of the works of the author and his co-authors on the application of nonlinear analysis methods for solving the nonlinear inverse problems (synthesis problems) for optimizing the different types of radiating systems are presented in the paper. The synthesis problem are formulated in variational statements and further they are reduced to research and numerical solution of nonlinear integral equations of Hammerstein type. The existence theorems are proof, the investigation methods of nonuniqueness problem of solutions and numerical algorithms of finding the optimal solutions are proved.
\end{abstract}

Keywords: nonlinear inverse problems, radiating systems, energy characteristics of directivity, nonlinear integral equations of the Hammerstein type, nonuniqueness and branching (or bifurcation) of solutions, numerical finding of optimal solutions, contour diagrams of directivity. 


\section{Introduction}

In many practical applications at the optimal design of various types of radio and acoustic radiating systems the requirements are only to the energy characteristics of the directivity of the radiated field (amplitude directivity pattern (DP) or DP by the power). Therefore there is a need to approximate real finite functions by modules of one-dimensional or twodimensional and discrete Fourier transform dependent on the real physical parameters. At the same time the absence of requirements to phase characteristics of field is used to improve the quality of approximation of synthesized DP to given.

Later on the variational formulations of different types of inverse problems in meansquare approach, which in further are reduced to investigation and numerical solution of onedimensional or two-dimensional nonlinear integral equations of the Hammerstein type with separate module and argument of desired complex-valued function, are considered. Nonuniqueness and branching (or bifurcation) of solutions dependent on the change of the physical parameters characterizing the radiating system are characteristic features of such equations. Problems on finding the set of branching points (bifurcation) are not investigated nonlinear one-parameter or two-parameter spectral problems.

The existence of connected components of the spectrum, which in the case of real parameters are of spectral lines, is essential difference between the two-dimensional and onedimensional spectral problems. The problem on finding the spectral lines is reduced to numerical solution of the Cauchy problem for an ordinary differential equation of the first order.

The degenerate of kernels in linear operators of the Hammerstein type equations is feature of the synthesis problems of antenna arrays. It allows to reduce nonlinear twoparameter spectral problems on finding the set of branching points of solutions to the corresponding systems of linear algebraic equations with nonlinear occurrence of the spectral parameters in the coefficients of system.

Iterative processes for numerical finding the various types of existing equations of the Hammerstein type equations (basic equations of synthesis) are proposed and justified, an analysis of their effectiveness is given. 


\section{Formulation of problems. Basic equations of synthesis}

In general case, the analysis problems (direct problems) of radio (or acoustic) radiating systems are reduced to solution the corresponding boundary problems of electrodynamics (acoustic) at a given excitation sources of fields [1-4] on the basis of Maxwell's equations (wave equation). The directivity pattern $\mathbf{f}$ is one of the basic characteristics of the emitted field on large distances from the radiating system. It describes the properties of the field in space dependent on the angular coordinates of a spherical coordinate system. In general case, the DP $\mathbf{f}$ is a vector of complex-valued function which has the form $[5,6]$

$$
\mathbf{f}=f_{\vartheta} \mathbf{i}_{\vartheta}+f_{\varphi} \mathbf{i}_{\varphi} \equiv A_{\vartheta} \mathbf{U} \mathbf{i}_{\theta}+A_{\varphi} \mathbf{U} \mathbf{i}_{\varphi} .
$$

Here $\mathbf{A}=\left\{A_{9}, A_{\varphi}\right\}$ is linear operator acting from some functional Hilbert space $H_{U}$ (the space of square integrable functions in the domain $V$, describing the distribution of extraneous fields (currents) $\mathbf{U}$ in volume $V$ ) into the space of complex-valued continuous functions $\mathbf{C}(\Omega)$ defined in some domain $\Omega \subseteq \mathbb{R}^{2}$ (or $\Omega \subseteq \mathbb{R}^{1}$ ) [7]. The form and properties of operators $A_{9}, A_{\varphi}$ are defined by type and geometry of the radiating system. The set (domain) of values of the operator $\mathbf{A}=\left\{A_{9}, A_{\varphi}\right\}$ is called [8,9] set or class of realized directivity patterns. This means that for any DP $\mathbf{f}$ from this class there exists such function of distribution of the currents (fields) $\mathbf{U} \in H_{U}$ that realizes this DP, i.e. $A \mathbf{U}=\mathbf{f}$.

In the simplest form the inverse problem (the synthesis problem) according to the prescribed amplitude DP can be formulated as the problem on finding the solutions of nonlinear operator equation of the first kind

$$
|A \mathbf{U}|=F,
$$

where $F$ is a given amplitude DP. In staged thus the synthesis problem all three correct conditions of problems by Hadamard [10-12]: existence of the solution, uniqueness of the solution, continuous dependence of the solution of the input data, can be violated simultaneously. Violation of condition 1) in the first place is connected with the fact that the given DP $F$ can not belong to the class realized, i. e. to the domain of values of the operator $A$. In other words, such DP can not be obtained at any distribution of field in the aperture of the radiating system belonging to the space $H_{U}$. Trying to recreate the DP $F$ just leads to effect of superdirectivity [5]. The system becomes resonance and critical to change of parameters. 
Condition 2) is violated due to the nonlinearity of the problem.

Therefore the variational formulations of problems, which in addition to the requirements of the basic characteristics of DP also contain requirements to the distribution function of the currents (fields) in the aperture of the radiating system, are considered. At that is required not complete coincidence obtained DP $|\mathbf{f}|$ with given $F$, but only the best (in the sense of the selected criterion) approximation to it. An important feature of the variational formulation of synthesis problems is the fact that in the optimization criterion can introduce functionals describing certain other requirements to amplitude-phase distribution (APD) of outside excitation sources. Their mean-square deviation, as a rule, will be used as the criterion of proximity of amplitudes of the given and synthesized DP.

\subsection{The case of linear polarization of extraneous field}

2.1.1. First we consider the scalar case of problems when extraneous fields (currents) in the radiating system is linearly polarized [7, 13], and created by their DP (2.1) has only one component. Let the operator $A$ acts from some Hilbert functional space $H_{U}=L_{2}(V)$ into the complex space of continuous and square integrable functions in domain $\Omega \subseteq \mathbb{R}^{2}$ (or $\Omega \subseteq \mathbb{R}^{1}$ ) $C_{(\Omega)}^{(2)}$.

In space $H_{U}$ we introduce the scalar product and norm

$$
\left(U_{1}, U_{2}\right)_{H_{I}}=\int_{V} U_{1}(P) \cdot \overline{U_{2}(P)} d V, \quad\|U\|_{H_{U}}=\left\{\int_{V}|U(P)|^{2} d V\right\}^{1 / 2},
$$

where $P=(x, y, z)$ is a point of integration.

Along with the Chebyshev norm

$$
\|f\|_{C(\Omega)}=\sup _{Q \in \Omega}|f(Q)|
$$

in the space we introduce scalar product and the generated by it norm and metric $C_{(\Omega)}^{(2)}$ as follows:

$$
(f, g)_{C_{(\Omega)}^{(2)}}=\int_{\Omega} f(Q) \overline{g(Q)} d Q, \quad\|f\|_{C_{(\Omega)}^{(2)}}=(f, f)_{C_{(\Omega)}^{(2)}}^{1 / 2}, \quad \rho_{C_{(\Omega)}^{(2)}}\left(f_{1}, f_{2}\right)=\left\|f_{1}-f_{2}\right\|_{C_{(\Omega)}^{(2)}}
$$

Note, space $C_{(\Omega)}^{(2)}$ is a Banach space relatively uniform norm (2.4) and it is incomplete space concerning norm defined according to (2.5) [14]. 
We consider also that the operator $A$ has the izometric property or it is completely continuous. Let the given amplitude DP $F$ is real positive (nonnegative) continuous function which different from nonzero in some limited closed domain $\bar{G} \subset \Omega$ and identically equal zero on complement $\Omega \backslash \bar{G}$. Let $A$ is isometric operator, i.e. for any $I \in H_{I}$ and $f=A U \in H_{f}$ equality is satisfied

$$
\|f\|_{C_{(\Omega)}^{(2)}}^{2}=(A U, A U)_{C_{(\Omega)}^{(2)}}=(U, U)_{H_{U}}=\|U\|_{H_{U}}^{2}
$$

In [15-17] the synthesis problem of given amplitude DP is investigated as a minimization problem in the Hilbert space $H_{U}=L_{2}(V)$ of the functional

$$
\sigma_{F}(U)=\|F-|A U|\|_{C_{(\Omega)}^{(2)}}^{2}
$$

characterizing the value of mean-square deviation of modules of given and synthesized DP in domain $\Omega$. For the formulated problem occurs [18-19]

Theorem 2.1. Let the linear operator $A: L_{2}(V) \rightarrow C_{(\Omega)}^{(2)}$ is isometric relatively meansquare metric and it is continuous operator from $L_{2}(V)$ into $C_{(\bar{G})}^{(2)}$ concerning uniform metric of the space $C_{(\Omega)}^{(2)}$, and the given amplitude $D P F(s)$ is a real positive (nonnegative) continuous function in the domain $\bar{G}$.

Then at least one point of absolute minimum of the functional (2.7) exists in the space $U=A^{*}\left(F \cdot e^{i \arg A U}\right)$ and a subsequence which converges weakly to one of points of absolute minimum can be selected from any minimizing sequence.

On the base of the necessary condition for an extremum of the functional (zero equality of its Hato differential [20]) $D \sigma_{F}(U, w)=\sigma_{F}^{\prime}(U)(w) \equiv 0$, we obtain the equation with respect to the optimal distribution of excitation sources

$$
U=A^{*}\left(F \cdot e^{i \arg A U}\right),
$$

where $A^{*}$ is the conjugate with $A$ operator.

Let the set of zeros $N(A)$ of the operator $A$ consists of only one zero element $\theta$. Then acting on both parts of (2.8) by operator $A$, we obtain the equivalent to (2.8) equation in space $C(\bar{G})$ :

$$
f=A A^{*}\left(F \cdot e^{i \arg f}\right) .
$$


By solutions $f_{*}$ of this equation the optimal distribution of excitation sources in radiating system are defined by the formula

$$
U_{*}=A^{*}\left(F \cdot e^{i \arg f_{*}}\right)
$$

From Theorem 2.1 and the properties of functional $\sigma_{F}(U)$ follows

Corollary 2.1. . Since functional $\sigma_{F}(U)$ is differentiable in $H_{I}$ by Hato, it is growing [21] and according to Theorem 2.1 has at least one point of absolute minimum, then (2.8) in the space $H_{U}$ and $(2.9)$ in the space $C(\bar{G})$ have at least one solution.

Lemma 2.1. Between solutions of (2.8) and (2.9) there exists bijection, that is if $U_{*}$ is the solution of (2.8), then $f_{*}=A U_{*}$ is the solution of (2.9). On the contrary, if $f_{*}$ is the solution of (2.9), then the corresponding solution of (2.8) is defined by (2.10).

The possibility of investigation of solutions of synthesis problems, using (2.8) or (2.9) follows from Lemma. Note, Equation (2.9) is simpler as (2.8), since the latter contains the operator exponent.

Note, solutions of synthesis problems according to the prescribed amplitude DP are determined with precision to value $e^{i \beta}$ ( $\beta$ is arbitrary constant), since $\left|U e^{i \beta}\right|=|U|$. So if there exists the solution of equations (2.8) (or (2.9)), then there is also generated by its family of solutions in which one solution different from another by phase constant. For the uniqueness of desired solutions additional conditions impose on the functions $\arg U$ or $\arg f$.

2.1.2. In the case completely continuous operator describing DP of radiating system the smoothing Tikhonov type functional [11]

$$
\sigma_{F_{\alpha}}(I)=\|F-|f|\|_{C_{(\bar{G})}^{(2)}}^{2}+\alpha\|U\|_{H_{I}}^{2} \equiv\|F-\mid A U\|_{C_{(\bar{G})}^{(2)}}^{2}+\alpha\|U\|_{H_{I}}^{2},
$$

which includes requirements as to the mean-square deviation of DP, so to norm of current, is used $[18,22]$ for the synthesis of various types of antennas. The parameter $\alpha$ can be viewed as regularization parameter $[11,23]$ or as a weighing coefficient, by means of which can control ratio between the first and second summand of functional.

Theorem 2.2. Let the linear operator A acts from the complex Hilbert space $H_{U}=L_{2}(V)$ into the complex space of continuous functions $C_{(\bar{G})}^{(2)}$ and it is completely 
continuous, and given DP is real positive (nonnegative) and continuous function in $\bar{G} \in \mathbb{R}^{1}$ (or in $\bar{G} \in \mathbb{R}^{2}$ ).

Then at least one point of absolute minimum of functional $\sigma_{F_{\alpha}}(U)$ exists in $H_{U}=L_{2}(V)$ and a subsequence which converges weakly to one of the points of absolute minimum can be selected from any minimizing sequence.

Differentiating functional $\sigma_{F_{\alpha}}(U)$ by Hato and performing appropriate transformations, we obtain the equation $[22,24]$

$$
\alpha U=-A^{*} A U+A^{*}(F \exp (i \arg (A U)))
$$

in the space $H_{U}$.

Equation with respect to synthesized DP based on equality $f=A U$ and (2.12) has the form

$$
\alpha f=-A A^{*} f+A A^{*}(F \exp (i \arg f)) .
$$

Lemma similar to Lemma 2.1 is valid for (2.12) and (2.13).

From Theorem 2.2 and properties of functional $\sigma_{F_{\alpha}}(U)$ follows $[7,18]$

Corollary 2.2. Since differentiable in $H_{U}$ by Hato functional $\sigma_{F_{\alpha}}(U)$ is growing and according to Theorem 2.2 has at least one point of absolute minimum, then (2.12) in space $H_{U}$ and (2.13) in the space $C(\bar{G})$ have at least one by one solution.

\subsection{The case of arbitrary polarization of excitation fields}

Consider the more general case when the excitation fields (or currents) in the radiating system and generated by it DP have vector character $[5,6]$. In this case, we set that the operator $A$ is completely continuous and it acts from $H_{U}=L_{2}(V) \otimes L_{2}(V) \otimes L_{2}(V)$ complex space of square integrable in the domain $V$ vector-valued functions, into the complex space of continuous functions on the compact $\bar{G} \subset \mathbb{R}^{2}$ vector-valued functions $\mathbf{C}_{(\bar{G})}^{(2)}=C_{(\bar{G})}^{(2)} \otimes C_{(\bar{G})}^{(2)}$ equipped by scalar product. We introduce the scalar product and generated by it norm in $H_{U}$ :

$$
\begin{aligned}
& \left(\mathbf{U}_{1}, \mathbf{U}_{2}\right)=\left(U_{x}^{(1)}, U_{x}^{(2)}\right)+\left(U_{y}^{(1)}, U_{y}^{(2)}\right)+\left(U_{z}^{(1)}, U_{z}^{(2)}\right) \equiv \\
& \equiv \int_{V}\left\{U_{x}^{(1)}(x, y, z) \overline{U_{x}^{(2)}(x, y, z)}+U_{y}^{(1)}(x, y, z) \overline{U_{y}^{(2)}(x, y, z)}++U_{z}^{(1)}(x, y, z) \overline{U_{z}^{(2)}(x, y, z)}\right\} d x d y d z
\end{aligned}
$$




$$
\|\mathbf{U}\|_{H_{I}}=(\mathbf{U}, \mathbf{U})^{1 / 2}=\left(\left(U_{x}, U_{x}\right)+\left(U_{y}, U_{y}\right)+\left(U_{z}, U_{z}\right)\right)^{1 / 2} .
$$

We define module of vector $\mathbf{U}$ as following: $|\mathbf{U}|=\left(U_{x} \cdot \overline{U_{x}}+U_{y} \cdot \overline{U_{y}}+U_{z} \cdot \overline{U_{z}}\right)^{1 / 2}$.

In the space $\mathbf{C}_{(\bar{G})}^{(2)}=C_{(\bar{G})}^{(2)} \otimes C_{(\bar{G})}^{(2)}$ along with the Chebyshev norm

$$
\|\mathbf{f}\|_{\mathbf{C}(\bar{G})}=\max _{(\vartheta, \varphi) \in \bar{G}}|\mathbf{f}(\vartheta, \varphi)|,
$$

where $|\mathbf{f}(\vartheta, \varphi)|=\left(f_{\vartheta}(\vartheta, \varphi) \cdot \overline{f_{\vartheta}(\vartheta, \varphi)}+f_{\varphi}(\vartheta, \varphi) \cdot \overline{f_{\varphi}(\vartheta, \varphi)}\right)^{1 / 2}$, we introduce the scalar product and generated by it the mean-square norm and metric

$$
\begin{gathered}
\left(\mathbf{f}_{1}, \mathbf{f}_{2}\right)_{\mathbf{C}_{(\bar{G})}^{(2)}}=\left(f_{\vartheta}^{(1)}, f_{\vartheta}^{(2)}\right)_{C_{(\bar{G})}^{(2)}}+\left(f_{\varphi}^{(1)}, f_{\varphi}^{(2)}\right)_{C_{(\bar{G})}^{(2)}} \equiv \iint_{\bar{G}}\left\{f_{\vartheta}^{(1)}(\vartheta, \varphi) \overline{f_{\vartheta}^{(2)}(\vartheta, \varphi)}+f_{\varphi}^{(1)}(\vartheta, \varphi) \cdot \overline{f_{\varphi}^{(2)}(\vartheta, \varphi)}\right\} \sin \vartheta d \vartheta d \varphi \\
\|\mathbf{f}\|_{\mathbf{C}_{(\bar{G})}^{(2)}}=(\mathbf{f}, \mathbf{f})_{\mathbf{C}_{(\bar{G})}^{(2)}}^{1 / 2}
\end{gathered}
$$

If DP of radiating system has two components $f_{\vartheta}, f_{\varphi}$, i.e. it is described by (2.1), then as the optimization criterions are used the following functionals [7]:

$$
\begin{gathered}
\sigma_{F_{\alpha}}(\mathbf{U})=\|F-|\mathbf{f}|\|_{C_{(\bar{G})}^{(2)}}^{2}+\alpha\|\mathbf{U}\|_{H_{I}}^{2} \equiv\|F-|A \mathbf{U}|\|_{C_{(\bar{G})}^{(2)}}^{2}+\alpha\|\mathbf{U}\|_{H_{I}}^{2}, \\
\tilde{\sigma}_{F_{\alpha}}(\mathbf{U})=\left\|F_{\vartheta}-\left|f_{\vartheta}\right|\right\|_{C_{(\bar{G})}^{(2)}}^{2}+\left\|F_{\varphi}-\left|f_{\varphi}\right|\right\|_{C_{(\bar{G})}^{(2)}}^{2}+\alpha\|\mathbf{U}\|_{H_{I}}^{2} \equiv\left\|F_{\vartheta}-\left|A_{\vartheta} \mathbf{U}\right|\right\|_{C_{(\bar{G})}^{(2)}}^{2}+\left\|F_{\varphi}-\left|A_{\varphi} \mathbf{U}\right|\right\|_{C_{(\bar{G})}^{(2)}}^{2}+\alpha\|\mathbf{U}\|_{H_{I}}^{2} .
\end{gathered}
$$

In the functional (2.20) $F_{9}, F_{\varphi}$, are the given amplitude of components of required DP. At that $F=\left(F_{\vartheta}^{2}+F_{\varphi}^{2}\right)^{1 / 2},\|F\|_{C(\bar{G})}=1$ and functions $F_{9}, F_{\varphi}$ can be given with account of existing requirements to polarization characteristics of emitted field.

If in the synthesis problem functional (2.19) is used as the optimization criterion, the problem on finding the minimum points is reduced to finding the solutions of the Euler equation

$$
\mathbf{U}=-\alpha^{-1} \mathbf{A}^{*} \mathbf{A} \mathbf{U}+\alpha^{-1} \mathbf{A}^{*}(F \mathbf{A} \mathbf{U} /|\mathbf{A} \mathbf{U}|)
$$

in the space $H_{I}$. Equivalent to (2.21) equation with respect to vector DP $\mathbf{f}$ in space $\mathbf{C}_{(\bar{G})}^{(2)}$ has the form

$$
\mathbf{f}=-\alpha^{-1} \mathbf{A} \mathbf{A}^{*} \mathbf{f}+\alpha^{-1} \mathbf{A A}^{*}(F \mathbf{f} /|\mathbf{f}|)
$$

In this case the following theorem is valid [7]. 
Theorem 2.3. Let linear completely continuous operator $A$ acts from the complex Hilbert space $H_{U}=L_{2}(V) \otimes L_{2}(V) \otimes L_{2}(V)$ into the complex space of continuous functions $\mathbf{C}_{(\bar{G})}^{(2)}=C_{(\bar{G})}^{(2)} \otimes C_{(\bar{G})}^{(2)}$ equipped by the scalar product, and given DP is a real positive continuous function on the compact $\bar{G}$.

Then in $H_{I}$ there exists at least one point of absolute minimum of functional $\sigma_{F_{\alpha}}(\mathbf{U})$ and a subsequence which converges weakly to one of the points of absolute minimum can select from any minimizing sequence.

For the functional (2.20) is true

Theorem 2.4. At conditions of Theorem 2.3 functional $\tilde{\sigma}_{F_{\alpha}}(\mathbf{U})$ in the space $H_{U}$ has at least one point of absolute minimum and subsequence which converges weakly to one of points of absolute minimum can be selected from any minimizing sequence.

For minimizing of the functional $\tilde{\sigma}_{F_{\alpha}}(\mathbf{U})$ in the space $H_{U}$ we obtain equation [7]

$$
\mathbf{U}=\alpha^{-1} \mathbf{A}^{*}\left\{\left(F_{\vartheta}-\left|A_{\vartheta} \mathbf{U}\right|\right) e^{i \arg A_{\vartheta} \mathbf{U}},\left(F_{\varphi}-\left|A_{\varphi} \mathbf{U}\right|\right) e^{i \arg A_{\varphi} \mathbf{U}}\right\} .
$$

Equivalent to (2.23) equation with respect to synthesized DP $\mathbf{f}$ in the space $\mathbf{C}_{(\bar{G})}^{(2)}$ has the form

$$
\mathbf{f}=\frac{1}{\alpha} \mathbf{A A}^{*}\left\{\left(F_{\vartheta}-\left|f_{\vartheta}\right|\right) e^{i \arg f_{\vartheta}},\left(F_{\varphi}-\mid f_{\varphi}\right) e^{i \arg f_{\varphi}}\right\}
$$

The existence of solutions of (2.23) in space $H_{U}$ and (2.24) in the space $\mathbf{C}_{(\bar{G})}^{(2)}$, respectively, follows from necessary condition of functional minimum $\tilde{\sigma}_{F_{\alpha}}(\mathbf{U})$.

If necessary the weight function $[15,17]$ can introduce in the functionals $\sigma_{F}$ and $\sigma_{F_{\alpha}}$ by means entering appropriate scalar products and affect on quality of the approximation of synthesized and given DP in a certain range of angles.

\subsection{Simultaneous optimization of the geometry of aperture and excitation fields}

The synthesis problems with optimization of geometry of radiating system are more complicated class of problems. These problems need to find a configuration of the radiating system and amplitude-phase distribution of excitation fields (currents) in it [25, 26]. Moreover, the operator $\mathbf{A}$ depends on two functions: function $\Gamma$ describing the geometry of the system, and amplitude-phase distribution function of excitation sources $\mathbf{U}$, i.e.

$$
\mathbf{f}=\mathbf{A}(\Gamma) \mathbf{U}
$$


In addition, the function $\mathbf{U}$ has, as rule, vector character, and the operator $\mathbf{A}$ is a nonlinear concerning the function $\Gamma$. Later on we shall consider the synthesis problem of a flat aperture, in which in addition to amplitude-phase distribution (APD) desired is too the function that describes the boundary of aperture. The basis of the formulation of such problems can be put the functionals (2.7), (2.11) and (2.20), expanding their by corresponding requirements to geometry of radiating system.

\subsection{Synthesis problem of discrete radiating systems - antenna arrays}

In many radio engineering systems antenna arrays (AR) have gained widespread use. Antenna array is $[4,6,27]$ antenna, which consists of $N$ identical (or different-type) radiators placing corresponding way in space and they collate by common system of power and control. In [28-37] investigations of nonlinear synthesis problems and planar antenna arrays according to the prescribed amplitude DP are presented.

To describe the electromagnetic characteristics of antenna arrays are used different by precision mathematical models [38-42]. In the base of construction of mathematical models is imposed $[40,42]$ that the excitation of each radiator is characterized by a unique complex number $I_{n}$ - complex amplitude of excitation. It's the physical meaning depends on the type of radiating system. On the base on the linearity of the Maxwell's equations the complex excitation amplitudes enter linearly in the expression for DP of array, i.e.

$$
\mathbf{f}(\vartheta, \varphi)=\sum_{n=1}^{N} I_{n} \mathbf{f}_{n}(\vartheta, \varphi) e^{i k\left(x_{n} \sin \vartheta \cos \varphi+y_{n} \sin \zeta \sin \varphi+z_{n} \cos \vartheta\right)},
$$

where $\mathbf{f}_{n}(\vartheta, \varphi)=f_{\vartheta}^{(n)}(\vartheta, \varphi) \mathbf{i}_{\vartheta}+f_{\varphi}^{(n)}(\vartheta, \varphi) \mathbf{i}_{\varphi}$ is vector DP $n$-th radiator. Vector $\mathbf{I}=\left\{I_{1}, I_{2}, \ldots, I_{N}\right\}$ is called vector excitation of array or vector of amplitude-phase distribution of currents in the array.

In general, the construction of high-accuracy mathematical models of array is reduced to solving the corresponding exterior boundary problem of high-frequency electrodynamics for system of the Maxwell's equations in multiply-connected domain [1, 39-41]. In the particular case, where the elements of the array are ideally leading talamy accounting of the mutual influence is based on the method of induced electromotive forces (IEF) and it is reduced to solution the corresponding system of linear integral equations [42]

$$
\mathrm{B} \mathbf{I}=\mathbf{U},
$$


where B is matrix-integral linear operator; $\mathbf{I}$ is complex-valued vector distribution function of surface currents on radiators; $\mathbf{U} \in H_{U}$ is vector-valued function describing the outside fields (voltage) which is necessary to create in the system of power of array. Allocating in the space $H_{I}$ compact class of solutions (where (2.27) is correct), solution of (2.27) is written as

$$
\mathbf{I}=\tilde{\mathrm{B}}^{-1} \mathbf{U}
$$

Here it is assumed that for the system of (2.27) exists the corresponding regularized system ${ }^{1}$ $\tilde{B} \mathbf{I}=\mathbf{U}$. Then on the basis of (2.28) formula for DP of arrray takes the form

$$
\mathbf{f}=A \tilde{\mathrm{B}}^{-1} \mathbf{U}
$$

This relation allows to formulate the synthesis problem of antenna array according to the prescribed amplitude DP with account of the mutual influence of elements as the problem on finding the vector $\mathbf{U}$ minimizing the functional

$$
\sigma_{F_{\alpha}}(\mathbf{U})=\left\|F-\left|A \tilde{\mathrm{B}}^{-1} \mathbf{U}\right|\right\|_{C_{(\bar{G})}^{(2)}}^{2}+\alpha\|\mathbf{U}\|_{H_{U}}^{2}
$$

in space $H_{U}$.

At need to take into account the component-wise approximation of modules of given and synthesized DP's, the functional [43-45]

$$
\begin{aligned}
& \tilde{\sigma}_{F_{\alpha}}(\mathbf{U})=\left\|F_{\vartheta}-\left|f_{\vartheta}\right|\right\|_{C_{(\bar{G})}^{(2)}}^{2}+\left\|F_{\varphi}-\left|f_{\varphi}\right|\right\|_{C_{(\bar{G})}^{(2)}}^{2}+\alpha\|\mathbf{U}\|_{H_{U}}^{2} \equiv \\
& \equiv\left\|F_{\vartheta}-\left|A_{9} \tilde{\mathrm{B}}^{-1} \mathbf{U}\right|\right\|_{C_{(\bar{G})}^{(2)}}^{2}+\left\|F_{\varphi}-\left|A_{\varphi} \tilde{\mathrm{B}}^{-1} \mathbf{U}\right|\right\|_{C_{(\bar{G})}^{(2)}}^{2}+\alpha\|\mathbf{U}\|_{H_{U}}^{2}
\end{aligned}
$$

analogously to (2.20) can be used as optimizing criterion.

By the desired solutions of this problem the optimal vector of extraneous voltages on inputs of radiators is determined on the basis of (2.27).

Thus, the basic requirements for synthesis problems of different types of radiating systems according to the prescribed amplitude DP are formulated. Note that recorded functionals is not convex [48], and therefore may have nonunique extreme point. In further the above statements of problems allow to obtain relatively simple nonlinear integral or matrix

\footnotetext{
${ }^{1}$ Note, regularization methods $[11,12]$ are the basic methods of construction of the operator $\widetilde{B}$ under which the stable solutions of (2.27) are obtained, and for equations whose kernels have a weak feature, - self-regularization. The entity of construction of the operator $\tilde{\mathrm{B}}$ (at execution of respective conditions) consists in reducing the system of integral equations of the first kind to the corresponding system of integral Fredholm equations of the second kind, i.e. to correct the problem.
} 
equations for the study and solution of which can be applied numerical methods of nonlinear analysis methods.

Integral equations method $[40,42]$ is used widely in such classes of problems. The method of synthesis of antenna arrays from cylindrical dipoles with account of mutual influence is proposed in [43-45]. Analysis of problem of nonuniqueness solutions is studied there by means computational experiments.

\subsection{Nonlinear synthesis problem of radiating systems with use of energy criterion}

In spite of the fact that from the amplitude DP $|\mathbf{f}|$ is easy to obtain the DP by power $N=|\mathbf{f}|^{2}$ and vice versa, in the mathematical aspect the synthesis problems of given amplitude DP $F_{0}$ and given energy DP $N_{0}=F_{0}^{2}$ are different tasks. For example, if $\left|\mathbf{f}_{*}\right|$ is the optimal solution of some variational synthesis problem of amplitude DP, then $\left|\mathbf{f}_{*}\right|^{2}$ will not be the optimal solution of a similar problem for the given DP $F_{0}^{2}$. On this basis, in [46-49] on the operator level are considered statements of synthesis problems with use of two types of stabilizing functionals, in which the vector character of the electromagnetic fields takes into account.

Consider the synthesis problem of given energy DP $N_{0}(\vartheta, \varphi)$. Taking into account (1.1.18) for DP by power

$$
N=|\mathbf{f}|^{2}=\left|f_{\vartheta}\right|^{2}+\left|f_{\varphi}\right|^{2} \equiv\left|A_{\vartheta} \mathbf{U}\right|^{2}+\left|A_{\varphi} \mathbf{U}\right|^{2},
$$

in the simplest form this problem can be formulated as a problem on finding the solutions of nonlinear operator equation of the first kind

$$
|\mathbf{A I}|^{2} \equiv\left|A_{\vartheta} \mathbf{U}\right|^{2}+\left|A_{\varphi} \mathbf{U}\right|^{2}=N_{0},
$$

where $N_{0}$ is a real positive continuous function in $\bar{G} \in \mathbb{R}^{2}$ (at that $\max _{(\vartheta, \varphi) \in \bar{G}} N_{0}(\vartheta, \varphi)=1$ ) which can not belong to the domain of values of the operator $|A \mathbf{U}|^{2}$. It is known [11] that (2.32) is severely ill-posed problem. In this connection, we consider the problem on best mean-square approximation of the real positive continuous (in the domain $\bar{G}$ ) function $N_{0}(\vartheta, \varphi)$ by function $|\mathbf{f}(\vartheta, \varphi)|^{2}\left(\mathbf{f}(\vartheta, \varphi)=A \mathbf{I} \in R(A), \mathbf{U}=\left\{U_{x}, U_{y}, U_{z}\right\} \in H_{U}\right)$. Formulate it as minimizing problem of functional [49]

$$
\sigma_{N_{\alpha}}(\mathbf{U})=\left\|N_{0}-|A \mathbf{U}|^{2}\right\|_{\mathbf{C}_{(\bar{G})}^{(2)}}^{2}+\alpha\|\mathbf{U}\|_{H_{I}}^{2} \equiv\left\|N_{0}-\left.\mathbf{f}\right|^{2}\right\|_{\mathbf{C}_{(\bar{G})}^{(2)}}^{2}+\alpha\|\mathbf{U}\|_{H_{I}}^{2}
$$


in the space $H_{U}$. In this functional the first summand characterizes mean-square deviation of given and synthesized DP by power. Second summand imposes restrictions on norm of currents in the radiating system. Real parameter $\alpha>0$ we shall consider as a weighing multiplier. The existence of at least one point of minimum functional $\sigma_{N_{\alpha}}(\mathbf{U})$ in the space $H_{U}$ states $[7,49]$

Theorem 2.5. Let the linear operator $A$ acts from the space $H_{U}$ into $\mathbf{C}_{(\bar{G})}^{(2)}$ and it is completely continuous, $N_{0}(\vartheta, \varphi)$ is given nonnegative continuous the function in $\bar{G}$, at that $\max _{(\vartheta, \varphi) \in \bar{G}} N_{0}(\vartheta, \varphi)=1$.

Then in the space $H_{U}$ there exists at least one point of absolute minimum of the functional $\sigma_{N_{\alpha}}(U)$ and subsequence that converges weakly to one of the points of absolute minimum can be selected from any minimizing sequence.

On the base of necessary condition of minimum functional is obtained the equation [7]

$$
\mathbf{U}=\frac{2}{\alpha} \mathbf{A}^{*}\left(N_{0} \cdot \mathbf{A U}\right)-\frac{2}{\alpha} \mathbf{A}^{*}\left(|\mathbf{A} \mathbf{U}|^{2} \cdot \mathbf{A U}\right)
$$

with respect to optimal currents in the space $H_{I}$. This equation is a nonlinear operator equation having in the right part linear operator along with the Hammerstein type operator. If the set of zeros $N(A)$ consists of only the zero element $\theta$, then acting on both parts of (2.34) by operator $A$, we obtain equation an equivalent to (2.34) with respect to synthesized DP in the space $\mathbf{C}_{(\bar{G})}^{(2)}$

$$
\mathbf{f}=\mathbf{B}(\mathbf{f}) \equiv \frac{2}{\alpha} \mathbf{A} \mathbf{A}^{*}\left(N_{0} \cdot \mathbf{f}\right)-\frac{2}{\alpha} \mathbf{A} \mathbf{A}^{*}\left(|\mathbf{f}|^{2} \cdot \mathbf{f}\right)
$$

In [49] is shown that the functional $\sigma_{N_{\alpha}}(\mathbf{U})$ has $m$-property [50], that is the minimum point of the functional is interior point of some convex weakly closed set of the space $H_{I}$. On this basis from Theorem 2.5 follows [7]

Corollary 2.3. Since the functional $\sigma_{N_{\alpha}}(\mathbf{U})$ is differentiable in $H_{I}$ by Hato, has at least one minimum point and $m$-property, then $(2.34)$ in the space $H_{I}$ and $(2.35)$ in the space $\mathbf{C}_{(\bar{G})}^{(2)}$ have at least by one solution.

Lemma 2.2. At conditions of Theorem 2.5 and limited values of parameter a operator 


$$
\mathbf{B}(\mathbf{f})=\frac{2}{\alpha} \mathbf{A A}^{*}\left(N_{0} \cdot \mathbf{f}\right)-\frac{2}{\alpha} \mathbf{A} \mathbf{A}^{*}\left(|\mathbf{f}|^{2} \cdot \mathbf{f}\right)
$$

is compact in the space $\mathbf{C}_{(\bar{G})}^{(2)}$.

Since for elements cocerning compact set of normalized space the strong and weak convergence coincide [51] then with Theorem 2.5 and Lemma 2.2 follows

Corollary 2.4. If $\left\{\mathbf{U}_{n}\right\}$ is minimizing sequence of the functional $\sigma_{N_{\alpha}}(\mathbf{U})$ converging weakly to the minimum point $\mathbf{U}_{*}$, then the sequence $\left\{\mathbf{f}_{n}=\mathbf{A U}_{n}\right\} \in \mathbf{C}_{(\bar{G})}^{(2)}$ converges uniformly in $\mathbf{C}_{(\bar{G})}^{(2)}$ to $\mathbf{f}_{*}=A \mathbf{I}_{*}$.

\section{About branching of solutions of the basic equations of synthesis. Partial cases}

Here on the example of scalar synthesis problems of linear radiator and radiating system with a flat aperture are presented the results of investigation of nonuniqueness problem of solutions corresponding to these tasks non-linear integral equations of Hammerstein type depending on the change of the physical parameters.

\subsection{The case of a linear radiator}

We put that the linear antenna is linear electric conductor of length $2 a$, sizes of cross-section of which are much less than the wavelength. Due to these limitations the excitation currents in the antenna shall have only direct-axis current [5]. Introduce the Cartesian and connected with spherical coordinate systems such that the origin of coordinates coincides with the middle of the antenna. We direct the axis $O Z$ along the antenna. Then the current vector in a Cartesian coordinate system will have only z-component $I(z)$. We shall introduce the dimensionless coordinates $s=\sin \theta / \sin \gamma_{0}, z^{\prime}=z / a$, and parameter

$$
c=k a \sin \gamma_{0}
$$

connecting the electric size of antenna with angle $2 \gamma_{0}$, outside of which given amplitude DP $F(s)$ identically zero. Then the formula for DP of linear antenna takes the form [7]

$$
f(s)=A U \equiv \int_{-1}^{1} U\left(z^{\prime}\right) e^{i c z^{\prime} s} d z^{\prime}
$$

For DP of antenna the Parseval equality [8] 


$$
\int_{-\infty}^{\infty}|f(s)|^{2} d s=(2 \pi / c) \int_{-1}^{1}\left|I\left(z^{\prime}\right)\right|^{2} d z^{\prime}
$$

is valid, that is the operator $A$ is isometric.

Taking into account that $F$ is finite function with compact carrier $\bar{G}=[-1,1]$ and expressions for the operators $A$ and $A^{*}$, we obtain the expanded form of (2.8):

$$
I(z)=(c / 2 \pi) \int_{-1}^{1} F(s) \exp \left\{i \arg \int_{-1}^{1} I\left(z^{\prime}\right) e^{i c z^{\prime} s} d z^{\prime}-i c z s\right\} d s .
$$

On the basis of (2.9) we obtain the Hammerstein type equation concerning optimal DP

$$
f(s)=B f \equiv \int_{-1}^{1} F\left(s^{\prime}\right) K\left(s, s^{\prime} ; c\right) e^{i \arg f\left(s^{\prime}\right)} d s^{\prime}
$$

in the space $C_{[-1,1]}^{(2)}$, where

$$
K\left(s, s^{\prime} ; c\right)=\frac{\sin c\left(s-s^{\prime}\right)}{\pi\left(s-s^{\prime}\right)} .
$$

The existence of at least one solution of (3.4) in the space $H_{I}=L_{2}[-1,1]$ and (3.5) in the space $C[-1,1]$ follows from Corollary 2.1 .

We shall present three important properties of (3.5).

$1^{\circ}$. If $f(s)$ is the solution of (3.5), then complex-conjugate function $\overline{f(s)}$ is the solution of (3.5) too.

$2^{\circ}$. If $f(s)$ is the solution of (3.5), then $e^{\beta} f(s)$ is the solution of (3.5) too, where $\beta$ is an arbitrary real constant.

$3^{\circ}$. For even functions $F(s)$ nonlinear operator $B$, which is in the right part of (3.5), transfers even phase DP in even, and odd - in an odd. That is the operator $B$ is invariant with respect to the type of parity of function $\arg f(s)$. Due to this property the existence of fixed points of the operator $B$ - solutions of (3.5) is possible in the classes of even and odd phase DP's.

In $[16,52]$ is shown that $(3.5)$ has two solutions in the class of real functions:

$$
f_{1}(s, c)=\int_{-1}^{1} F\left(s^{\prime}\right) K\left(s, s^{\prime} ; c\right) d s^{\prime},
$$

which is called the primary solution of the first type and

$$
f_{2}(s, c)=\int_{-1}^{1} F\left(s^{\prime}\right) K\left(s, s^{\prime} ; c\right) \operatorname{sgn}\left(s^{\prime}-s_{0}\right) d s^{\prime}
$$


is the primary solution of the second type. Point $s_{s} s_{0}(c)$ is determined from the condition $f_{2}(s, c)=0$. For the even $F(s) \quad s_{0}=0$, that is the solution (3.8) is a real odd function (the corresponding to it amplitude DP is even function).

These solutions are effective only at small values of parameter $c$. With the growth of this parameter there exist branching points $c_{i}$ at which more effective (in the sense value of functional $\sigma_{F}$ ) complex solutions branch-off from real solutions.

Consider according to $[7,16]$ results of investigation of branchings of primary solution of the first type of solutions of (3.5). Using the procedure of decomplexification of the space $C[-1,1][14]$ from (3.5) we move to the equivalent system

$$
\left\{\begin{array}{l}
u(s)=\int_{-1}^{1} Q_{1}\left[s, s^{\prime}, c, u\left(s^{\prime}\right), v\left(s^{\prime}\right)\right] d s^{\prime} \equiv \int_{-1}^{1} F\left(s^{\prime}\right) K\left(s, s^{\prime} ; c\right) \frac{u\left(s^{\prime}\right)}{\sqrt{u^{2}\left(s^{\prime}\right)+v^{2}\left(s^{\prime}\right)}} d s^{\prime}, \\
v(s)=\int_{-1}^{1} Q_{2}\left[s, s^{\prime}, c, u\left(s^{\prime}\right), v\left(s^{\prime}\right)\right] d s^{\prime} \equiv \int_{-1}^{1} F\left(s^{\prime}\right) K\left(s, s^{\prime} ; c\right) \frac{v\left(s^{\prime}\right)}{\sqrt{u^{2}\left(s^{\prime}\right)+v^{2}\left(s^{\prime}\right)}} d s^{\prime}
\end{array}\right.
$$

On the base of the branching theory of solutions [53] the problem on finding such values of parameter $c=c_{l}$ (branching points) and all different from $f_{1}\left(s, c_{l}\right)$ solutions of system of (3.9) satisfying the conditions

$$
\left\{\begin{array}{l}
\max _{s \in[-1,1]}\left|u\left(s, c_{l}\right)-f_{1}\left(s, c_{l}\right)\right| \rightarrow 0, \\
\max _{s \in[-1,1]}\left|v\left(s, c_{l}\right)\right| \rightarrow 0,
\end{array} \quad \text { at } c \rightarrow c_{l},\right.
$$

are considered. Condition (3.10) means that it is necessary to find small continuous solutions $w(s)=u(s, c)-f_{1}\left(s, c_{l}\right), \omega(s)=v(s, c)$, converging uniformly to zero at $c \rightarrow c_{1}$. Putting in (3.9)

$$
u(s, c)=f_{1}\left(s, c_{l}\right)+w(s), \quad v(s)=0+\omega(s) .
$$

and expanding the integrand $Q_{1}, Q_{2}$ in the vicinity of the point $\left(c_{1}, f_{1}\left(s, c_{1}\right), 0\right)$ in the power series by $w, \omega$ and $\mu$, and taking into account that the function $f_{1}(s, c)$ is its solution, we obtain system of integral equations of Lyapunov-Schmidt type with respect to small solutions $W(s), \omega(s)$ :

$$
\begin{aligned}
& w(s)=a\left(s, c_{l}\right) \mu+\sum_{m+n+p \geq 2}^{\infty} \mu^{p} \int_{-1}^{1} A_{m n p}\left(s, s^{\prime}\right) w^{m}\left(s^{\prime}\right) \omega^{n}\left(s^{\prime}\right) d s^{\prime}, \\
& \omega(s)-\int_{-1}^{1} F\left(s^{\prime}\right) K\left(s, s^{\prime} ; c\right) \frac{\omega\left(s^{\prime}\right)}{f_{1}\left(s^{\prime}, c\right)} d s^{\prime}=\sum_{m+n+p \geq 2}^{\infty} \mu^{p} \int_{-1}^{1} B_{m m p}\left(s, s^{\prime}\right) w^{m}\left(s^{\prime}\right) \omega^{n}\left(s^{\prime}\right) d s^{\prime},
\end{aligned}
$$


where $a\left(s, c_{1}\right)=\int_{-1}^{1} A_{001}\left(s, s^{\prime}\right) d s^{\prime}$. On the base of the left part of (3.13) we obtain linear homogeneous integral equation

$$
\omega(s)=T(c) \omega \equiv \int_{-1}^{1} F\left(s^{\prime}\right) K\left(s, s^{\prime} ; c\right) \frac{\omega\left(s^{\prime}\right)}{f_{1}\left(s^{\prime}, c\right)} d s^{\prime}
$$

for finding the points of possible branching of solutions.

Equation (3.14) is a nonlinear one-parameter spectral problem concerning parameter $c$. It is shown in $[7,16]$ that for a given even amplitude DP $F(s)$ there exist branching points of two types: eigenvalues of multiplicity two correspond to the first type, eigenvalues the multiplicity of three - to the second type. It is found in [54] analytical expressions for eigenfunctions of (3.14) and are obtained systems of transcendental equations for finding the possible branching points.

Using for finding the solutions of branching equation the Newton diagram method, it is shown [53] that two complex-conjugate between themselves solutions, which in the first approximation, have the form

$$
f_{1,2}^{(1)}\left(s, c_{1}^{(l)}+\mu\right)=f_{1}\left(s, c_{1}^{(l)}\right)+\left(a\left(s, c_{1}^{(l)}\right)+\alpha_{020}^{(1)}\left(s, c_{1}^{(l)}\right) h_{1}^{2}\right) \mu \pm i \varphi_{2}\left(s, c_{1}^{(l)}\right) h_{1} \mu^{1 / 2}+O\left(\mu^{3 / 2}\right)
$$

branch-off from the real solution $f_{1}(s)$ in the branching points of the first type $c_{1}^{(l)}$. Here $a\left(s, c_{1}^{(l)}\right), \alpha_{020}^{(1)}\left(s, c_{1}^{(l)}\right)$ are even by $s$ functions which are obtained by means corresponding transformations, $\varphi_{2}\left(s, c_{1}^{(l)}\right)=s \cdot f_{1}\left(s, c_{1}^{(l)}\right)$ is the second eigenfunction of (3.14) at the points $c_{1}^{(l)}$,

It is shown [7] also that the branching-off solutions branch-off too.

Analogous investigations [7] are conducted in branching points of the second type $c_{2}^{(l)}$.

To estimate the effectiveness of different types of solutions we consider value of the functional $\sigma_{F}$ depending on the parameter $c$, which it takes on these solutions. For example, in Fig. 3.1 are shown the values of the functional for $F(s)=1$. The most effective solution images envelope which: on the segments I corresponds to the primary solution $f_{1}$, on II branching-off solutions $f_{1,2}^{(1)}$ at the point $c_{1}^{(1)}$ with odd phase DP, on III - solutions $f_{1,2}^{(1)}$ and branching-off solutions $\widetilde{f}_{1,2}^{(1)}$ from these, on IV - solutions $\tilde{f}_{1,2}^{(1)}$ and branching-off 


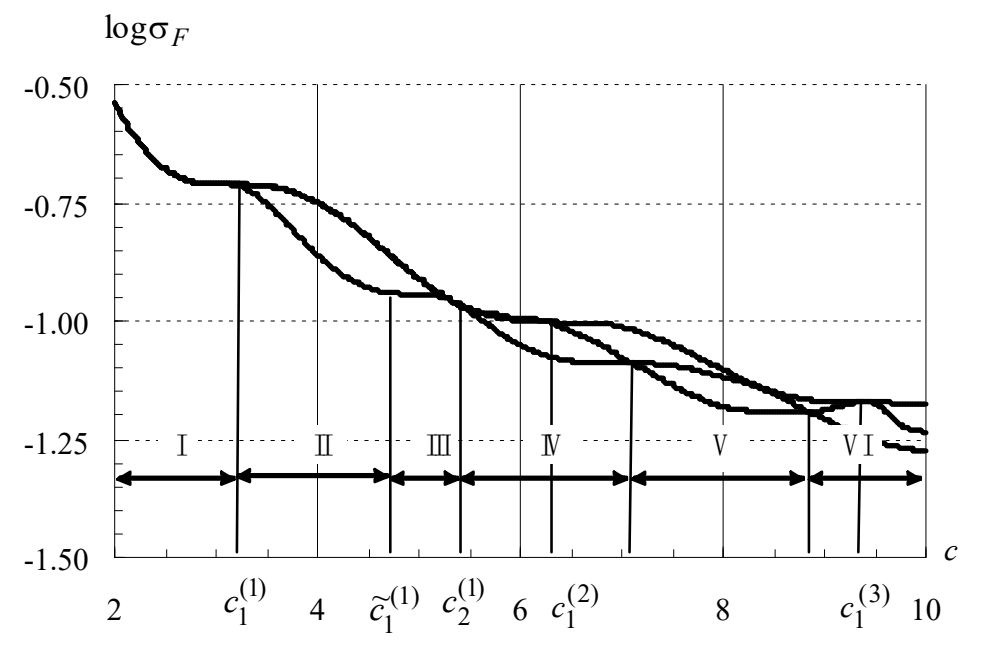

Fig 3.1. The value of the functional $\sigma_{F}$ on the first primary

and branching-off solutions for $F(s)=1$

solutions $f_{1,2}^{(2)}$ at the point $c_{2}^{(1)}$ with even phase, на $\mathrm{V}$ - branching-off at the point $c_{1}^{(2)}$ solutions of the type $f_{1,2}^{(1)}$, on VI - solutions $f_{1,2}^{(2)}$.

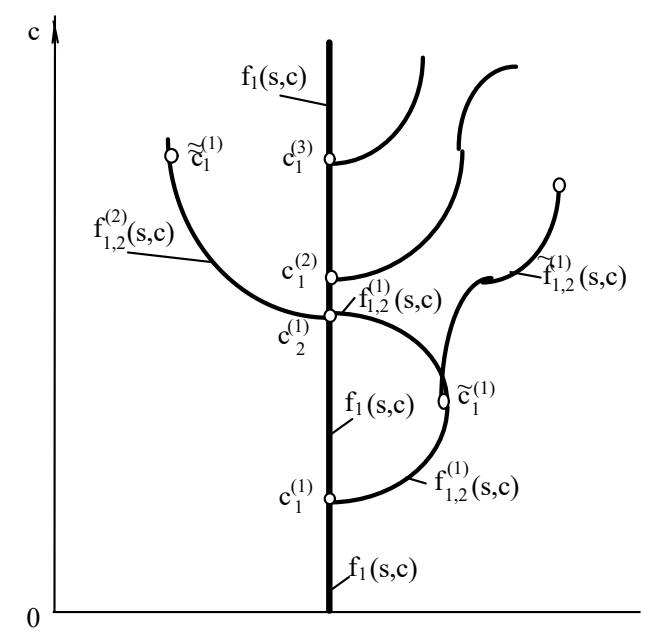

Fig. 3.2. "Tree" of solutions

Thus, the analytical investigations $[7,16,52]$ and the results of numerical experiments enable to describe the general structure of the solutions of the problem dependeing on change of the value of the parameter $c$.

Because the values of the functional on some types of solutions in a given interval of change of the parameter $c$ may be equall, the curves shown in Fig. 3.1, does not map the full 
structure of the existing solutions. For greater clarity this structure can be represented schematically as a "tree" of solutions. In Fig. 3.2 it is shown for the case of even DP. The primary solution $f_{1}(s, c)$ is central. The solution $f^{(1)}(s, c)$ with odd phase DP $\left(\arg f^{(1)}(s, c)\right)$ branches first. At the point $\widetilde{c}_{1}^{(1)}$ solution $\widetilde{f}^{(1)}(s, c)$ branches from branching solution $f^{(1)}(s, c)$, and solution $f^{(1)}(s, c)$ enter in a real solution in a neighborhood of the point $c_{2}^{(1)}$ at $c$, tending to $c_{2}^{(1)}$. At the same point $c_{2}^{(1)}$ solution with even phase DP $f^{(2)}(s, c)$ branches from primary solution. The solution of the type $f^{(1)}(s, c)$ with an odd phase DP branches at the point $c_{1}^{(2)}$, which is located directly behind $c_{2}^{(1)}$. The solutions of the types $f^{(1)}(s, c)$ and $f^{(2)}(s, c)$ form basic branches of "tree". The possible branching points of branching-off solutions are shown on these branches.

\subsection{Radiating system with a flat aperture}

3.2.1. Basic equations and relations. Consider according with [55-58] the synthesis problems of a flat aperture assuming that form of aperture $S$ is known and a field has elliptical polarization. Let the plane in which aperture is located, coincides with the plane $X O Y$ of the Cartesian coordinate system. Then the radiated field in the far zone can be represented by the formula [6]:

$$
\mathbf{E}(R, \vartheta, \varphi)=-\frac{k}{4 \pi} \frac{e^{-i k R}}{R} \mathbf{D}(\vartheta, \varphi),
$$

where

$\mathbf{D}(\vartheta, \varphi)=\left[\mathbf{i}_{r} \times\left[\left(\mathbf{n}-\mathbf{i}_{r}\right) \times \mathbf{f}\right]\right]$,

$$
\mathbf{f}(\vartheta, \varphi)=\iint_{S} \mathbf{U}(x, y) e^{i k(x \sin \vartheta \cos \varphi+y \sin \vartheta \sin \varphi)} d x d y,
$$

$\mathbf{i}_{r}$ is radial ort of spherical coordinate system, $\mathbf{n}=-\mathbf{i}_{z}, \mathbf{f}(\vartheta, \varphi)$ is a vector DP of flat aperture $S$. Since $\mathbf{n}=-\mathbf{i}_{z}$, function $\mathbf{U}(x, y)$ describes the tangential component of the electric vector $\mathbf{E}$ or vector of current flowing through the aperture $S$ :

$$
\mathbf{U}(x, y)=U_{x}(x, y) \mathbf{i}_{x}+U_{y}(x, y) \mathbf{i}_{y} .
$$

Introducing in a far zone special coordinate system [6] $\mathbf{g}_{1}, \mathbf{g}_{2}, \mathbf{g}_{3}$ connected with orts of spherical coordinate system by formulas

$$
\mathbf{g}_{1}=\cos \varphi \mathbf{i}_{\vartheta}-\sin \varphi \mathbf{i}_{\varphi}, \quad \mathbf{g}_{2}=\sin \varphi \mathbf{i}_{\vartheta}+\cos \varphi \mathbf{i}_{\varphi}, \quad \mathbf{g}_{3}=\mathbf{i}_{r},
$$


enables the vector synthesis problem to reduce to two independent scalar synthesis problems.

Obviously, the system $\mathbf{g}_{1}, \mathbf{g}_{2}, \mathbf{g}_{3}$ is orthonormal, and transformation (3.18) is rotation of spherical coordinate system on an angle $\varphi$ around the vector $\mathbf{i}_{r}$. At that vector $\mathbf{D}(\vartheta, \varphi)$ in the coordinate system (3.18) has the form [6]

$$
\mathbf{D}=(1+\cos \vartheta) \cdot\left[f_{x} \mathbf{g}_{1}+f_{y} \mathbf{g}_{2}\right] \text {, }
$$

where

$$
f_{x, y}(\vartheta, \varphi)=A_{x, y} U_{x, y} \equiv \iint_{S} U_{x, y}(x, y) e^{i k(x \sin \vartheta \cos \varphi+y \sin \vartheta \sin \varphi)} d x d y .
$$

For mappings (3.20) the Parseval equality [85]:

$$
\left\|A_{x, y} U_{x, y}\right\|_{H_{f}}^{2}=\left\|U_{x, y}\right\|_{H_{U}}^{2}
$$

are valid, that is operators $A_{x}, A_{y}$ are isometric.

Consider the synthesis problem of a flat aperture, in which component-wise deviation of modules given and synthesized diagrams is taken into account. As optimization criterion we choose the functional type

$$
\begin{gathered}
\sigma_{F}(\mathbf{U})=\iint_{G}\left\{\left[F_{g_{1}}\left(s_{1}, s_{2}\right)-\left|f_{g_{1}}\left(s_{1}, s_{2}\right)\right|\right]^{2}+\left[F_{g_{2}}\left(s_{1}, s_{2}\right)-\left|f_{g_{2}}\left(s_{1}, s_{2}\right)\right|\right]^{2}\right\} d s_{1} d s_{2}+ \\
+\iint_{\mathbb{R}^{2} \backslash \bar{G}}\left\{\left|f_{g_{1}}\left(s_{1}, s_{2}\right)\right|^{2}+\left|f_{g_{2}}\left(s_{1}, s_{2}\right)\right|^{2}\right\} d s_{1} d s_{2},
\end{gathered}
$$

where $F_{g_{1}}, F_{g_{2}}$ are modulus of components of the given amplitude DP $F=\left(F_{g_{1}}^{2}+F_{g_{2}}^{2}\right)^{1 / 2}$ in closed domain $G \in \mathbb{R}^{2}$. This criterion provides not only proximity of modules of given $F$ and synthesized $|\mathbf{f}|$ DP's, but it allows certain to influence on the polarization characteristics of the radiated field [5]. On the base of the necessary minimum condition of the functional (3.22) and the corresponding transformation we obtain system equations (these equations is not connected between themselves) concerning components of synthesized DP:

$$
f_{g_{j}}\left(s_{1}, s_{2}\right)=B_{j} f_{g_{j}} \equiv\left(\frac{k}{2 \pi}\right)^{2} \iint_{\bar{G}} F_{g_{j}}\left(s_{1}^{\prime}, s_{2}^{\prime}\right) K\left(s_{1}, s_{2}, s_{1}^{\prime}, s_{2}^{\prime} ; k\right) e^{i \arg f_{g_{j}}\left(s_{1}^{\prime}, s_{2}^{\prime}\right)} d s_{1}^{\prime} d s_{2} \quad(j=1,2),
$$

where

$$
K\left(s_{1}, s_{2}, s_{1}^{\prime}, s_{2}^{\prime} ; k\right)=\iint_{S} e^{i k\left[x\left(s_{1}-s_{1}^{\prime}\right)+y\left(s_{2}-s_{2}^{\prime}\right)\right]} d x d y
$$


is a kernel. In the case of rectangular aperture the kernel $K\left(s_{1}, s_{2}, s_{1}^{\prime}, s_{2}^{\prime} ; c_{1}, c_{2}\right)$ takes the form

$$
K\left(s_{1}, s_{2}, s_{1}^{\prime}, s_{2}^{\prime} ; c_{1}, c_{2}\right)=\frac{\sin c_{1}\left(s_{1}-s_{1}^{\prime}\right)}{c_{1}\left(s_{1}-s_{1}^{\prime}\right)} \cdot \frac{\sin c_{2}\left(s_{2}-s_{2}^{\prime}\right)}{c_{2}\left(s_{2}-s_{2}^{\prime}\right)},
$$

where

$$
c_{1}=k a_{1} \sin \gamma_{1}, \quad c_{2}=k a_{2} \sin \gamma_{2}
$$

are real numeric parameters characterizing the sizes of aperture $a_{1}, a_{2}$ in wavelengths, $k=2 \pi / \lambda$ is wave number, $\gamma_{1}, \gamma_{2}$ are angles that characterize the domain $\bar{G}$ (solid angle), in which different from the identity components of amplitude DP $F_{g_{j}}\left(s_{1}^{\prime}, s_{2}^{\prime}\right)$ are given.

Later on we omit index in (3.23) and shall investigate the solutions of one equation

$$
f(Q)=B f \equiv \iint_{\bar{G}} F\left(Q^{\prime}\right) K\left(Q, Q^{\prime}, \mathbf{c}\right) e^{i \arg f\left(Q^{\prime}\right)} d Q^{\prime},
$$

where for reduction of records we introduce the following notations

$$
Q=\left(s_{1}, s_{2}\right), \quad d Q^{\prime}=d s_{1}^{\prime} d s_{2}^{\prime}, \quad \mathbf{c}=\left(c_{1}, c_{2}\right) .
$$

Thus, the synthesis problem of flat radiating system with arbitrary polarization of irradiation according to the prescribed amplitude DP is reduced to two independent and simpler synthesis problems with linearly polarized fields in the aperture.

Equation (3.27) is a nonlinear two-dimensional integral equation of the Hammerstein type and it has nonunique solutions. Their quality and properties depend on the form of aperture $S$, the values of parameters $c_{1}, c_{2}$ and properties of given amplitude DP $F$.

On the base of decomplexification [138] we shall consider the complex space $\mathbf{C}(\bar{G})$ as a direct sum of two real spaces of continuous functions $\mathbf{C}(\bar{G})=C(\bar{G}) \oplus C(\bar{G})$ in the domain $\bar{G}$. The elements of this space are written as: $f=(u, v) \in \mathbf{C}(\bar{G}), u=\operatorname{Re}(f) \in C(\bar{G}), v=\operatorname{Im}(f) \in C(\bar{G})$. Norms in these spaces have the form:

$$
\|u\|_{C(\bar{G})}=\max _{Q \in \bar{G}}|u(Q)|, \quad\|v\|_{C(\bar{G})}=\max _{Q \in \bar{G}}|v(Q)|,\|f\|_{C(\bar{G})}=\max \left(\|u\|_{C(\bar{G})},\|v\|_{C(\bar{G})}\right)
$$

Equation (3.27) in the decomplexified space $\mathbf{C}(G)$ is reduced to equivalent to it system of equations 


$$
\begin{aligned}
& u(Q)=B_{1}(u, v) \equiv \iint_{\bar{G}} F\left(Q^{\prime}\right) K\left(Q, Q^{\prime}, \mathbf{c}\right) \frac{u\left(Q^{\prime}\right)}{\sqrt{u^{2}\left(Q^{\prime}\right)+v^{2}\left(Q^{\prime}\right)}} d Q^{\prime}, \\
& v(Q)=B_{2}(u, v) \equiv \iint_{\bar{G}} F\left(Q^{\prime}\right) K\left(Q, Q^{\prime}, \mathbf{c}\right) \frac{v\left(Q^{\prime}\right)}{\sqrt{u^{2}\left(Q^{\prime}\right)+v^{2}\left(Q^{\prime}\right)}} d Q^{\prime} .
\end{aligned}
$$

Denote the closed convex set of continuous functions as $S_{M} \subset \mathbf{C}(\bar{G})$ setting that

$$
\begin{aligned}
& S_{M}=S_{M_{u}} \oplus S_{M_{v}}, \quad S_{M_{u}}=\left\{u \in S_{M_{u}}:\|u\|_{C(\bar{G})} \leq M\right\}, \\
& S_{M_{v}}=\left\{v \in S_{M_{v}}:\|v\|_{C(\bar{G})} \leq M\right\}, \quad M=\max _{Q \in \bar{G}} \iint_{\bar{G}} F\left(Q^{\prime}\right)\left|K\left(Q, Q^{\prime}, \mathbf{c}\right)\right| d Q .
\end{aligned}
$$

Consider one of the properties of the function $\exp \left(i \arg f\left(Q^{\prime}\right)\right)$ that included in (3.26) at $f\left(Q^{\prime}\right) \rightarrow 0$. Obviously,

$$
\exp \left(i \arg f\left(Q^{\prime}\right)\right)=\frac{f\left(Q^{\prime}\right)}{\left|f\left(Q^{\prime}\right)\right|} \equiv \frac{u\left(Q^{\prime}\right)+i v\left(Q^{\prime}\right)}{\left(u^{2}\left(Q^{\prime}\right)+v^{2}\left(Q^{\prime}\right)\right)^{1 / 2}}
$$

is a continuous function if $u\left(Q^{\prime}\right)=\operatorname{Re} f\left(Q^{\prime}\right)$ and $v\left(Q^{\prime}\right)=\operatorname{Im} f\left(Q^{\prime}\right)$ are continuous functions, at that $\left|\exp \left(i \arg f\left(Q^{\prime}\right)\right)\right|=1$ for any $f\left(Q^{\prime}\right)$. If $u\left(Q^{\prime}\right) \rightarrow 0$ and $v\left(Q^{\prime}\right) \rightarrow 0$ simultaneously, then $f\left(Q^{\prime}\right) \equiv 0$ is a complex zero with undefined argument by definition [60, p. 20]. On this basis at $u\left(Q^{\prime}\right) \rightarrow 0$ and $v\left(Q^{\prime}\right) \rightarrow 0$ we redefine $\exp \left(i \arg f\left(Q^{\prime}\right)\right)$ as function, module of which is equal to one and argument is undefined.

Theorem 3.1. The operator $\mathbf{B}=\left(B_{1}, B_{2}\right)^{T}$ determined by the formulas (3.29) maps a closed convex set $S_{M}$ of the Banach space $\mathbf{C}(\bar{G})$ in itself and it is completely continuous.

As the corollary from the Theorem 3.1 follows satisfaction of conditions of the Schauder principle [14, p. 411] according to which the operator $\mathbf{B}=\left(B_{1}, B_{2}\right)^{T}$ has a fixed point $f_{*}=\left(u_{*}, v_{*}\right)^{T}$ belonging to the set $S_{M}$. This point is a solution of a system of (3.29) and (3.27), respectively.

Easily to be convinced that function

$$
f_{0}(Q, \mathbf{c})=\iint_{\bar{G}} F\left(Q^{\prime}\right) K\left(Q, Q^{\prime}, \mathbf{c}\right) d Q^{\prime}
$$

is one of solutions of (3.27) in the case of symmetric domain $\bar{G}$.

In $[56,57]$ it is shown that the operator 


$$
D f \equiv \iint_{\bar{G}} K\left(Q, Q^{\prime}, \mathbf{c}\right) f\left(Q^{\prime}\right) d Q^{\prime}
$$

is positive on the cone of nonnegative functions $\mathrm{K}$ of the space $C(\bar{G})$ [61]. According to this the operator $D$ leaves invariant cone $\mathrm{K}$, i.e. $D \mathrm{~K} \subset \mathrm{K}$. Since $F \subset \mathrm{K}$, the primary solution $f_{0}=D F$ is also nonnegative function in the fdomain $\bar{G}$.

To find the branching lines and complex solutions of (3.27) that branch-off from the real (primary) solution $f_{0}(Q, \mathbf{c})$, we shall consider the problem on finding such a set of parameter values $\mathbf{c}^{(0)}=\left(c_{1}^{(0)}, c_{2}^{(0)}\right)$ and all different from $f_{0}(Q, \mathbf{c})$ solutions of (3.29) that at $\left|\mathbf{c}-\mathbf{c}^{(0)}\right| \rightarrow 0$ satisfy the conditions

$$
\max _{Q \in \bar{\Omega}}\left|u(Q, \mathbf{c})-f\left(Q, \mathbf{c}^{(0)}\right)\right| \rightarrow 0, \quad \max _{Q \in \bar{\Omega}}|v(Q, \mathbf{c})| \rightarrow 0 .
$$

These conditions indicate the need to find such small continuous in $G$ solutions,

$$
w(Q, \mathbf{c})=u(Q, \mathbf{c})-f_{0}\left(Q, \mathbf{c}^{(0)}\right), \quad \omega(Q, \mathbf{c})=v(Q, \mathbf{c}),
$$

which converge uniformly to zero as $\mathbf{c} \rightarrow \mathbf{c}^{(0)}$. At that it should take into account also the direction of movement of vector $\mathbf{c}$ to vector $\mathbf{c}^{(0)}$.

Set $c_{1}=c_{1}^{(0)}+\mu, \quad c_{2}=c_{2}^{(0)}+v$, and desired solutions we find in the form

$$
u(Q, \mathbf{c})=f_{0}\left(Q, \mathbf{c}^{(0)}\right)+w(Q, \mu, v), \quad v(Q, \mathbf{c})=\omega(Q, \mu, v) .
$$

We write the system of nonlinear integral equations of Lyapunov-Schmidt with respect to small solutions $w, \omega$ as

$$
\begin{aligned}
& u(Q)=a_{10}\left(Q, \mathbf{c}^{(0)}\right) \mu+a_{01}\left(Q, \mathbf{c}^{(0)}\right) \mathcal{v} \\
& +\sum_{m+n+p+q \geq 2} \mu^{p} v^{q} \iint_{\Omega} A_{m n p q}\left(Q, Q^{\prime}, \mathbf{c}^{(0)}\right) w^{m}\left(Q^{\prime}\right) \omega^{n}\left(Q^{\prime}\right) d Q^{\prime}, \\
& \omega(Q)-\iint_{\bar{\Omega}} F(Q) K\left(Q, Q^{\prime}, \mathbf{c}^{(0)}\right) \frac{\omega\left(Q^{\prime}\right)}{f_{0}\left(Q^{\prime}, \mathbf{c}^{(0)}\right)} d Q^{\prime}= \\
& =\sum_{m+n+p+q \geq 2} \mu^{p} v^{q} \iint_{\bar{\Omega}} B_{m n p q}\left(Q, Q^{\prime}, \mathbf{c}^{(0)}\right) w^{m}\left(Q^{\prime}\right) \omega^{n}\left(Q^{\prime}\right) d \Omega_{Q^{\prime}} .
\end{aligned}
$$

Here $A_{\text {mирq }}\left(Q, Q^{\prime}, \mathbf{c}^{(0)}\right), B_{\text {mирq }}\left(Q, Q^{\prime}, \mathbf{c}^{(0)}\right)$ are coefficients of expansion of integrand functions of (3.29) in uniform convergent power series. 
The problem on finding the set of possible branching points of solutions of (3.35) and (3.36) is reduced [56] to find the eigenvalues of two-dimensional linear homogeneous integral equation

$$
\varphi(Q)=T\left(c_{1}, c_{2}\right) \varphi \equiv \iint_{\Omega} \frac{F\left(Q^{\prime}\right)}{f_{0}\left(Q^{\prime}, c_{1}, c_{2}\right)} K\left(Q, Q^{\prime}, c_{1}, c_{2}\right) \varphi\left(Q^{\prime}\right) d Q^{\prime}
$$

at condition $f_{0}\left(Q^{\prime}, \mathbf{c}\right)>0$. Eigenfunctions of $(3.37)$ are used $[56,57]$ at the construction of branching-off solutions of (3.35) and (3.36).

3.2.2. Nonlinear two-parameter spectral problem. Note that (3.37) in the general case is a nonlinear two-parameter spectral problem. For the numerical finding the approximate solutions it is necessary to construct its digitization and consider the corresponding problem in finite-dimensional spaces. It should be noted that in the literature, in particular in [62, 63], more attention is given to the construction of numerical methods for solving the nonlinear one-parameter problems.

In [64-66] a general method for finding the approximate solutions of (3.37), which may be applicable to a wide range of nonlinear two-parameter spectral problems is proposed.

Denote the spectral parameters as $\lambda=\left(\lambda_{1}, \lambda_{2}\right)$. Let $E$ and $V$ are complex Banach spaces, and the vector parameter $\lambda=\left(\lambda_{1}, \lambda_{2}\right)$ belongs to domain (open connected set) $\Lambda=\Lambda_{1} \times \Lambda_{2}$ of the complex space $\mathbb{C}^{2}=\mathbb{C} \times \mathbb{C}$, where $\lambda_{i} \in \Lambda_{i} \subset \mathbb{C}, \Lambda_{i}=\left\{\lambda_{i} \in \Lambda_{i}:\left|\lambda_{i}\right|<r_{\lambda}\right\} \quad(i=1,2), r_{\lambda}$ is some real constant. Consider the operator-function $\mathrm{A}(\cdot, \cdot): \Lambda \rightarrow \mathrm{L}(E, V)$, where to every $\lambda=\left(\lambda_{1}, \lambda_{2}\right) \in \Lambda$ is put in correspondence operator $\mathrm{A}\left(\lambda_{1}, \lambda_{2}\right) \in \mathrm{L}(E, V)$. Here the space of linear bounded operators [14] is marked as $\mathrm{L}(E, V)$.

We shall consider the nonlinear two-parameter spectral problem of the form

$$
\mathrm{A}\left(\lambda_{1}, \lambda_{2}\right) x=0
$$

where necessary to find the eigenvalues $\lambda=\left(\lambda_{1}^{(0)}, \lambda_{2}^{(0)}\right) \in \Lambda$ and corresponding eigenvectors $x^{(0)} \in E\left(x^{(0)} \neq 0\right)$ such that $\mathrm{A}\left(\lambda_{1}^{(0)}, \lambda_{2}^{(0)}\right) x^{(0)}=0$.

Let the Banach spaces $E$ and $E_{n}, n=1,2, \ldots$, be given and also a system $\mathrm{P}=\left(p_{n}\right)_{n \in \mathbb{N}}$ of linear bounded operators $p_{n}: E \rightarrow E_{n}$ such that

$$
\left\|p_{n} x\right\|_{E_{n}} \rightarrow\|x\|_{E} \quad(n \in \mathbb{N}) \quad \forall x \in E .
$$


Operators $p_{n}$ are called connecting $[14,68]$. Note, by the principle of uniform boundedness [14] with (3.37) follows inequality $\left\|p_{n}\right\| \leq$ const . Let in every space $E_{n}$ the element $x_{n}$ be selected. Writing these elements in order to increase the numbers we shall form a sequence $\left\{x_{n}\right\}$.

Let element $x_{n}$ is selected in each space $E_{n}$. Writing these elements in ascending numerical order, we form sequence $\left\{x_{n}\right\}$.

Definition 3.1 [68]. The sequence $\left\{x_{n}\right\}_{n \in \mathbb{N}^{\prime}}$ from $x_{n} \in E_{n} \quad \mathrm{P}$-converges (discrete converges) to $x \in E$ if $\left\|x_{n}-p_{n} x\right\|_{E_{n}} \rightarrow 0\left(n \in \mathbb{N}^{\prime}\right)$; we denote $x_{n} \stackrel{\mathrm{P}}{\longrightarrow} x\left(n \in \mathbb{N}^{\prime}\right)$.

Definition of different types of convergence of operators $A_{n}$ to $A$ is given in [68]. Later on only required in further definition of stable convergence ${ }^{2}{ }_{n}$ to $\mathrm{A}$ is presented.

Discretization of initial problem (3.38), $\mathrm{t}$ the choice of the space $E_{n}$ and definition of operators $p_{n}: E \rightarrow E_{n}$ can be differentially. In particular, one of the approaches to the digitization of (3.38) if the operator-function $\mathrm{A}\left(\lambda_{1}, \lambda_{2}\right)$ is described by formula A $\left(\lambda_{1}, \lambda_{2}\right) \equiv\left(T\left(\lambda_{1}, \lambda_{2}\right)-I\right)$, where $T\left(\lambda_{1}, \lambda_{2}\right)$ is linear continous operator, $I$ is unique ort in the separable (infinite-dimensional) Hilbert space $E$, consist in following. Take an arbitrary complete orthonormal system of functions $\left\{x_{k}\right\}_{k=1}^{\infty}$ in $E$. Each element $x \in E$ is represented as a series $x=\sum_{k=1}^{\infty} c_{k} x_{k}$, where $c_{k}=\left(x, x_{k}\right)$ is Fourier coefficient of element $x$. Since $T\left(\lambda_{1}, \lambda_{2}\right)$ is linear continuous operator acting in separable Hilbert space, it admits the matrix representation [69]:

$$
T_{M}\left(\lambda_{1}, \lambda_{2}\right)=\left(t_{j k}\left(\lambda_{1}, \lambda_{2}\right)\right)_{j, k=1}^{\infty},
$$

where $t_{j k}\left(\lambda_{1}, \lambda_{2}\right)=\left(T\left(\lambda_{1}, \lambda_{2}\right) x_{k}, x_{j}\right)$. At that sequence of the Fourier coefficients of element $y=T\left(\lambda_{1}, \lambda_{2}\right) x$ is obtained from the sequence of Fourier coefficients of element $x$ by means transformation matrix $T_{M}\left(\lambda_{1}, \lambda_{2}\right)$.

Using the matrix representation of the operator $T_{M}\left(\lambda_{1}, \lambda_{2}\right)$ the spectral problem (3.38) is formulated as

\footnotetext{
${ }^{2}$ Definition 3.2 [68]. The sequance $\left\{A_{n}\right\}_{n \in \mathbb{N}}$ of operators $A_{n} \in L\left(E_{n}, V_{n}\right)$ converges stably to $A \in L(E, V)$, if $A_{n} \stackrel{\mathrm{P} Q}{\longrightarrow} A$ $(n \in \mathbb{N})$ and the following condition (stability condition) is valid: $A_{n}^{-1} \in L\left(V_{n}, E_{n}\right)$ exists at all $n$, at that $\left\|A_{n}^{-1}\right\| \leq$ const $\left(n \geq n_{0}\right)$.
} 


$$
\mathrm{A}_{M}\left(\lambda_{1}, \lambda_{2}\right) x \equiv\left(T_{M}\left(\lambda_{1}, \lambda_{2}\right)-I_{M}\right) x=0
$$

where $I_{M}$ is identity matrix in the space of sequences $l_{2}$. Thus, the operators $T(\lambda)$ and $T_{M}(\lambda)$ are equivalent in the sense that they put in correspondence one and the same element $y \in E$, but we obtain the Fourier coefficients of element $y=T(\lambda) x$ as a result of operation of operator $T_{M}(\lambda)$ on element $x$. Obviously, that the spectrums of these operators coincide, i.e. the spectral problem (3.41) and the problem

A $\left(\lambda_{1}, \lambda_{2}\right) x \equiv\left(T\left(\lambda_{1}, \lambda_{2}\right)-I\right) x=0$

are equivalent.

According to $[14,68]$ applying to the problem (3.38) other discretization methods, including the following: quadrature (cubature) processes in the case of homogeneous integral equations and changing the derivatives by difference analogues in differential equations, we obtain the approximation problems for approximate finding the eigenvalues and eigenfunctions in finite-dimensional spaces

$$
\mathrm{A}_{n}\left(\lambda_{1}, \lambda_{2}\right) x_{n}=0 \quad n \in \mathbb{N}
$$

At that the problem on finding the eigenvalues is reduced to finding the roots of the $n$-th order determinant, i.e. the roots of the equation

$$
\Psi_{n}\left(\lambda_{1}, \lambda_{2}\right) \equiv \operatorname{det}\left\|a_{i, j}^{(n)}\left(\lambda_{1}, \lambda_{2}\right)\right\|_{i, j=1}^{n}=0 \quad(n \in \mathbb{N}) .
$$

Consider the necessary in further auxiliary one-parameter spectral problem as a particular case of (3.38). Set that variable $\lambda_{2}$ in the operator-function $A\left(\lambda_{1}, \lambda_{2}\right)$ is expressed by some unique differentiable function $\lambda_{2}=z\left(\lambda_{1}\right)$ mapping domain $\Lambda_{1, \beta} \subseteq \Lambda_{1}$ in some subdomain $\Lambda_{2, \beta} \subset \Lambda_{2}$. In the simplest case we put $\lambda_{2}=\beta \lambda_{1}$, where $\beta$ is a real parameter. Introduce into consideration at $\lambda_{1} \in \Lambda_{1, \beta}$ operator function $A_{\beta}\left(\lambda_{1}\right) \equiv A\left(\lambda_{1}, z\left(\lambda_{1}\right)\right.$ ) (narrowing of operator-function $\left.\mathrm{A}\left(\lambda_{1}, \lambda_{2}\right)\right)$. One-parameter nonlinear spectral problem

$$
\mathrm{A}_{\beta}\left(\lambda_{1}\right) x=0
$$

is connected with it. Here to each value $\lambda=\left(\lambda_{1}, z\left(\lambda_{1}\right)\right) \in \Lambda$ operator $\mathrm{A}_{\beta}\left(\lambda_{1}, z\left(\lambda_{1}\right)\right) \in \mathrm{L}(E, V)$ is put in correspondence.

Analogously to (3.42)we consider approximating sequence of discretizing problems (3.44) at $n \in \mathbb{N}$ 


$$
\mathrm{A}_{\beta, n}\left(\lambda_{1}, z\left(\lambda_{1}\right)\right) x_{n}=0, \quad n \in \mathbb{N} .
$$

The spectrum of operator-function $\mathrm{A}_{\beta}\left(\lambda_{1}\right)$ is denoted as $s\left(\mathrm{~A}_{\beta}\right)$. Suppose that $s\left(\mathrm{~A}_{\beta}\right) \neq \Lambda_{1, \beta}$. For spectral $s(\mathrm{~A})$ of $(3.368)$ holds $[56,67]$

Theorem 3.2. Let the following conditions be satisfied:

1) operator-function $\mathrm{A}(\cdot, \cdot): \Lambda \rightarrow \mathrm{L}(E, V)$ is holomorphic, and $s(\mathrm{~A}) \neq \Lambda$;

2) operator-functions $\mathrm{A}_{n}(\cdot, \cdot): \Lambda \rightarrow \mathrm{L}(E, V)$ are holomorphic and for any closed bounded set $\Lambda_{0} \subset \Lambda$ the following inequality $\max _{\lambda \in \Lambda_{0}}\left\|\mathrm{~A}_{n}\left(\lambda_{1}, \lambda_{2}\right)\right\| \leq c\left(\Lambda_{0}\right)=$ const $(n \in \mathbb{N})$ is valid;

3) operators $\mathrm{A}\left(\lambda_{1}, \lambda_{2}\right) \in \mathrm{L}(E, V), \mathrm{A}_{n}\left(\lambda_{1}, \lambda_{2}\right) \in \mathrm{L}\left(E_{n}, V_{n}\right) \quad(n \in \mathbb{N})$ are the Fredholm operators with zero index for any $\lambda=\left(\lambda_{1}, \lambda_{2}\right) \in \Lambda$;

4) spectrum $s\left(\mathrm{~A}_{\beta}\right) \neq \Lambda_{1, \beta}$ and a sequence of functions $\Psi_{n}\left(\lambda_{1}, \lambda_{2}\right)$ are differentiable in the domain $\Lambda$;

5) $\mathrm{A}_{n}(\lambda) \rightarrow \mathrm{A}(\lambda)$ is stable for any $\lambda \in r(\mathrm{~A})=\Lambda \backslash s(\mathrm{~A})$.

Then the following statements are true:

1) every point of spectrum $\lambda_{1}^{(0)} \in s\left(\mathrm{~A}_{\beta}\right)$ is isolated, it is eigenvalue of the operator $\mathrm{A}_{\beta}\left(\lambda_{1}\right) \equiv \mathrm{A}\left(\lambda_{1}, z\left(\lambda_{1}\right)\right)$, the finite-dimensional eigensubspace $N\left(\mathrm{~A}\left(\lambda_{1}^{(0)}\right)\right)$ and the finitedimensional root subspace correspond to it;

2) for each $\lambda_{1}^{(0)} \in s\left(\mathrm{~A}_{\beta}\right)$ there exists a sequence $\left\{\lambda_{1, n}^{(0)}\right\}$ from $\lambda_{1, n}^{(0)} \in s\left(\mathrm{~A}_{\beta, n}\right) \quad\left(n>n_{0}\right)$, such that $\lambda_{1, n}^{(0)} \rightarrow \lambda_{1}^{0}$

3) each point $\lambda^{(0)}=\left(\lambda_{1}^{(0)}, z\left(\lambda_{1}^{(0)}\right)\right) \in \Lambda$ is a spectrum point of the operator-function $\mathrm{A}\left(\lambda_{1}, \lambda_{2}\right)$;

4) if in some small $\varepsilon_{0}$ - neighborhood of the point $\lambda^{(0)}=\left(\lambda_{1}^{(0)}, z\left(\lambda_{1}^{(0)}\right)\right) \in \Lambda$ at all $n$ larger any number $N_{0}$ (corresponding $\varepsilon_{0}$, according to definition of limit of sequence p. 2)) the sequence of partial derivates $\left\{\frac{\partial \Psi_{n}}{\partial \lambda_{2}}\left(\lambda_{1, n}^{0}, z\left(\lambda_{1, n}^{0}\right)\right)\right\}$ is nonzero, then in an arbitrarily small $\varepsilon_{*}$ neighborhood of point $\left(\lambda_{1}^{(0)}, z\left(\lambda_{1}^{(0)}\right)\right) \in \Lambda$ there exists a continuous differentiable function $\lambda_{2, N_{*}}=\varphi_{N_{*}}\left(\lambda_{1}\right)$, which is solution of (3.53), at that $\lambda_{2, N_{*}}^{(0)}=\varphi_{N_{*}}\left(\lambda_{1, N_{*}}^{(0)}\right)$ and at the point 
$\left(\lambda_{1, N_{*}}^{(0)}, \lambda_{2, N_{*}}^{(0)}\right)=\left(\lambda_{1, N_{*}}^{(0)}, \varphi_{N_{*}}\left(\lambda_{1, N_{*}}^{(0)}\right)\right)$ however little differs from point of a spectrum of auxiliary oneparameter problem (3.55) $\left|\lambda_{1, N_{*}}^{(0)}-\lambda_{1}^{(0)}\right|<\varepsilon_{*} ;$ i.e. in some bicylindrical domain $\Lambda_{0}=\left\{\left(\lambda_{1}, \lambda_{2}\right) \in \Lambda_{0}:\left|\lambda_{1}-\lambda_{1}^{(0)}\right|<\varepsilon_{1},\left|\lambda_{2}-\lambda_{2}^{(0)}\right|<\varepsilon_{2}\right\}$ there exists a connected component of spectrum of the operator-function $\mathrm{A}_{N_{*}}\left(\lambda_{1}, \lambda_{2}\right)\left(\varepsilon_{1}, \varepsilon_{2}\right.$ are small real constants).

Proof. The proof of the theorem is given in [56] and is based on theorems 1 and 2 with $[68$, p. 68,69$]$ and the theorem about existence of implicit function (see, for example, [70]).

If the points $\left(\lambda_{1, v}^{(0)}, \lambda_{2, v}^{(0)}\right) \in \Lambda$ are the eigenvalues of (3.42) and derivatives $\partial \Psi_{n} / \partial \lambda_{1}$, $\partial \Psi_{n} / \partial \lambda_{2}$ in these points are nonzero, to find connected components of the spectrum of this problem on the base of (3.43) Cauchy problem [56, 57]we solve the in a neighborhood of each point $\left(\lambda_{1, v}^{(0)}, \lambda_{2, v}^{(0)}\right) \in \Lambda$

$$
\begin{aligned}
& \frac{d \lambda_{2}}{d \lambda_{1}}=-\frac{\partial \Psi_{n}\left(\lambda_{1}, \lambda_{2}\right) / \partial \lambda_{1}}{\partial \Psi_{n}\left(\lambda_{1}, \lambda_{2}\right) / \partial \lambda_{2}}, \\
& \lambda_{2}\left(\lambda_{1, v}^{(0)}\right)=\lambda_{2, v}^{(0)} .
\end{aligned}
$$

3.2.3. Numerical algorithms for finding the possible branching lines of solutions. Return to finding the solutions of (3.37), in which $c_{1}, c_{2}$ are spectral parameters. Let $\left(c_{1}, c_{2}\right) \in \Lambda_{c}$, $\Lambda_{c}=\Lambda_{c_{1}} \times \Lambda_{c_{2}}$, where $\Lambda_{c_{i}}=\left\{c_{i} \in \Lambda_{c_{i}}: 0<c_{i}<r_{c}\right\}$. By direct check we set that for arbitrary values of the parameters $\left(c_{1}, c_{2}\right) \in \Lambda_{c}$ the function

$$
\varphi_{0}(Q, \mathbf{c})=\iint_{\bar{\Omega}} F\left(Q^{\prime}\right) K\left(Q, Q^{\prime}, \mathbf{c}\right) d Q^{\prime}=f_{0}(Q, \mathbf{c})
$$

is one of the eigenfunctions, i.e. there exists a connected set of the spectrum, coinciding with the domain $\Lambda_{c}$. As a result of this, the condition $s(\tilde{A}) \neq \Lambda_{c}$ is not satisfied. To find another connected components of spectrum we exclude eigenfunction (3.37) from the kernel of integral equation, namely, consider the equation

$$
\varphi(Q, \mathbf{c})=\tilde{T}(\mathbf{c}) \varphi \equiv \iint_{\Omega} \tilde{K}\left(Q, Q^{\prime}, \mathbf{c}\right) \varphi\left(Q^{\prime}\right) d Q^{\prime},
$$

where

$$
\tilde{K}\left(Q, Q^{\prime}, \mathbf{c}\right)=\frac{F\left(Q^{\prime}\right)}{f_{0}\left(Q^{\prime}, \mathbf{c}\right)} K\left(Q, Q^{\prime}, \mathbf{c}\right)-\frac{\psi_{0}(Q) \varphi_{0}\left(Q^{\prime}, \mathbf{c}\right)}{\left\|\psi_{0}\right\|\left\|\varphi_{0}\right\|} .
$$


Here $\psi_{0}(Q)$ is adjoint with (3.37) eigenfunction of equation of From Lemma Schmidt [53, p. 132] follows that from spectrum of operator $\tilde{T}(\mathbf{c})$ is excluded coherent component coinciding with the domain $\Lambda_{c}$ and the corresponding to the function $\varphi_{0}(Q, \mathbf{c})$.

Using to (14) certain convergent cubature process with coefficients $a_{j n} \in \mathbb{R}$ and nodes $Q_{j n} \in \bar{\Omega} \quad(n \in \mathbb{N})$ and rejecting in it remainder, we obtain homogeneous system of linear algebraic equations (SLAE)

$$
u_{i n}=T_{M_{n}}\left(c_{1}, c_{2}\right) \mathbf{u} \equiv \sum_{j=1}^{n} a_{j n} \tilde{\mathrm{K}}\left(Q_{i n}, Q_{j n}, c_{1}, c_{2}\right) u_{j n}(i=1 \div n),
$$

where $u_{i n}=u\left(Q_{i n}\right)$.

The presence of such values of parameters $c_{1}, c_{2}$, which are the solutions of the equation

$$
\Psi_{n}\left(c_{1}, c_{2}\right)=\operatorname{det}\left(T_{M_{n}}\left(c_{1}, c_{2}\right)-I_{n}\right)=0,
$$

is necessary condition of the existence different from zero solutions of (3.51). We consider (3.52) as the problem on finding the implicitly given function $c_{2}=\gamma\left(c_{1}\right)$, reducing it to the Cauchy problem (3.46) and (3.47). Putting $c_{2}=\beta c_{1}$ in (3.49), we shall consider the auxiliary one-parameter spectral problem

$$
\varphi(Q)=\tilde{\tilde{T}}\left(c_{1}\right) \varphi \equiv \iint_{\bar{\Omega}} \tilde{\tilde{K}}\left(Q, Q^{\prime}, c_{1}\right) \varphi\left(Q^{\prime}\right) d Q^{\prime},
$$

solutions of which we use as initial conditions in the Cauchy problem (3.47). Corresponding this equation SLAE has the form

$$
u_{i n}=\tilde{T}_{M_{n}}\left(c_{1}\right) u_{n} \equiv \sum_{j=1}^{n} a_{j n} \tilde{K}\left(Q_{i n}, Q_{j n}, c_{1}\right) u_{j n} \quad(i=1, \ldots, n)
$$

and the problem on finding the eigenvalues of this system is reduced to finding the roots of the equation $\tilde{\Psi}_{n}\left(c_{1}\right)=\operatorname{det}\left(\tilde{\tilde{T}}_{M_{n}}\left(c_{1}\right)-I_{n}\right)=0$. For the numerical solution of the Cauchy problem (3.46) and (3.47) are used the Runge-Kutta and Adams methods.

We shall present numerical examples of finding the solutions of (3.37) for two given amplitude DP's. It is shown in Fig. 3.3 spectral lines of (3.37) corresponds the given DP $F\left(s_{1}, s_{2}\right) \equiv 1$ and in Fig. 3.4 - given DP, which is defined by the formula: 


$$
F\left(s_{1}, s_{2}\right)= \begin{cases}2 \sqrt{\left(s_{1}^{2}+s_{2}^{2}\right)} \cdot \sqrt{\left(1-\left(s_{1}^{2}+s_{2}^{2}\right)\right)}, & \left(s_{1}^{2}+s_{2}^{2}\right) \leq 1, \\ 0, & \left(s_{1}^{2}+s_{2}^{2}\right)>1\end{cases}
$$

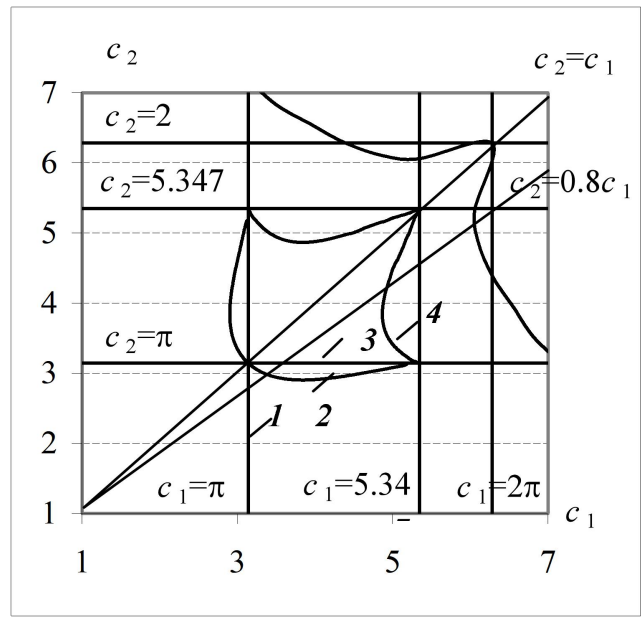

Fig. 3.3. The possible branching lines of solutions of system of (3.29) for $F\left(s_{1}, s_{2}\right) \equiv 1$

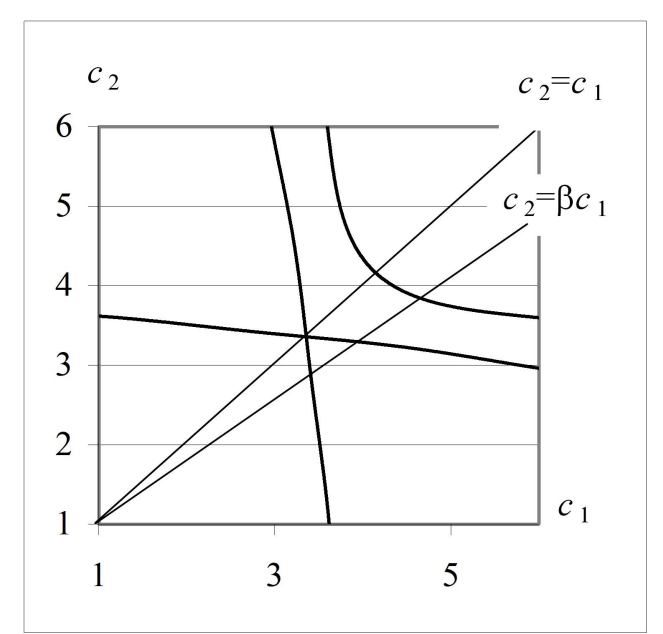

Fig. 3.4. The possible branching lines of solutions of system of (3.29) for $F\left(s_{1}, s_{2}\right)$, which is defined by (3.54)

3.2.4. Variational approach to solution of the nonlinear spectral problems. In $[71,72]$ along with the implicit functions method a variational approach to solution of the nonlinear one-parameter and two-parameter spectral problems on finding the eigenvalues $\lambda=\left\{\lambda_{1}, \lambda_{2}\right\} \in \Lambda_{c} \subset \mathbb{R}^{2}$ and eigenelements $u \in U \subset L_{2}(\Omega)$ of equation

$$
T(\lambda) u=u
$$

in the real Hilbert space $L_{2}(\Omega)$ for the case when $T(\lambda): L_{2}(\Omega) \rightarrow L_{2}(\Omega)$ is a linear positive definite self-adjoint operator nonlinearly depending on the parameters $\lambda_{1}, \lambda_{2}$, is proposed. Variational problem is formulated as the problem on finding such values of parameter $\lambda_{*} \in \Lambda_{c}$ and such functions $u_{*} \in L_{2}(\Omega)$ on which functional

$$
\varphi(\lambda, u)=\|T(\lambda) u-u\|_{L_{2}}^{2} \equiv(T(\lambda) u-u, T(\lambda) u-u)
$$

becomes minimum. The equivalence of the spectral problem (3.55) and put it in correspodence of variational problem (3.56) is proved. Based on the method of generalized 
coordinate descent iterative process for the numerical finding one of the eigenvalues and the corresponding eigenfunction of (3.55) is suggested. Local convergence is proved.

Example of use of a variational approach to finding the eigenvalues and eigenfunctions of (3.37) is shown in Fig. 3.5 for the case when the function $F\left(s_{1}, s_{2}\right)$ is defined by (3.54). Later on the eigenfunctions of (3.37), corresponding to eigenvalues belonging to curves 1,3 illustrated in Fig. 3.5,b, are shown. From the analysis of the figures we see that the eigenfunctions $\varphi_{1}\left(s_{1}, s_{2}\right)$ are odd by argument $s_{1}$ and functions $\varphi_{2}\left(s_{1}, s_{2}\right)$ are odd by both arguments.

Found by numerical method form and properties of eigenfunctions in the possible branching points are used to determine of the properties of branching-off in these points of solutions of nonlinear systems of (3.29).

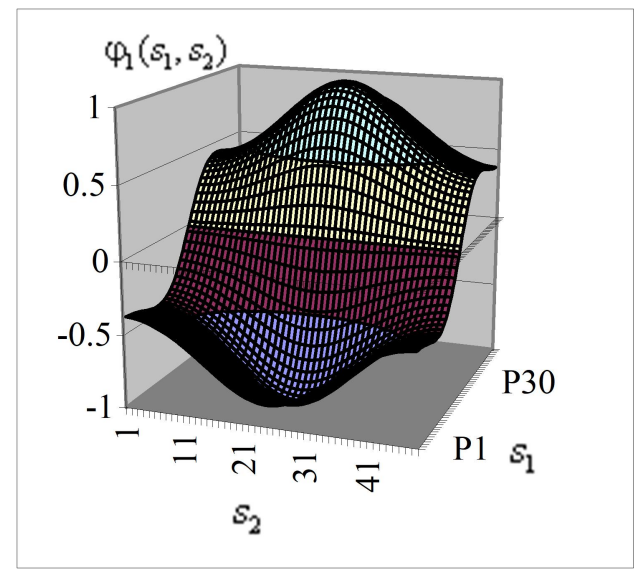

$a$

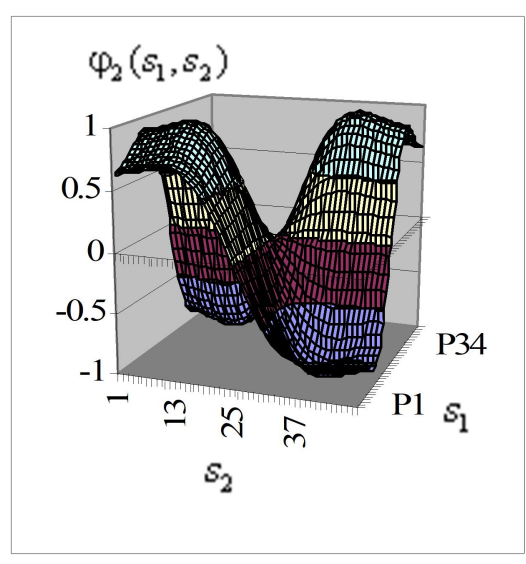

$b$

Fig. 3.5. Normalized eigenfunctions of $(3.88)$ corresponding to the eigenvalues: $a-(\mathrm{c} 1=\mathbf{3 . 1 4 1 5 9 3 9 3 3 1 1}, \mathrm{c2}=2.51327514648), b-(\mathrm{c} 1=$ 3.64391021729, $c 2=\mathbf{2 . 9 1 5 1 2 8 1 7 3 8 3 )}$
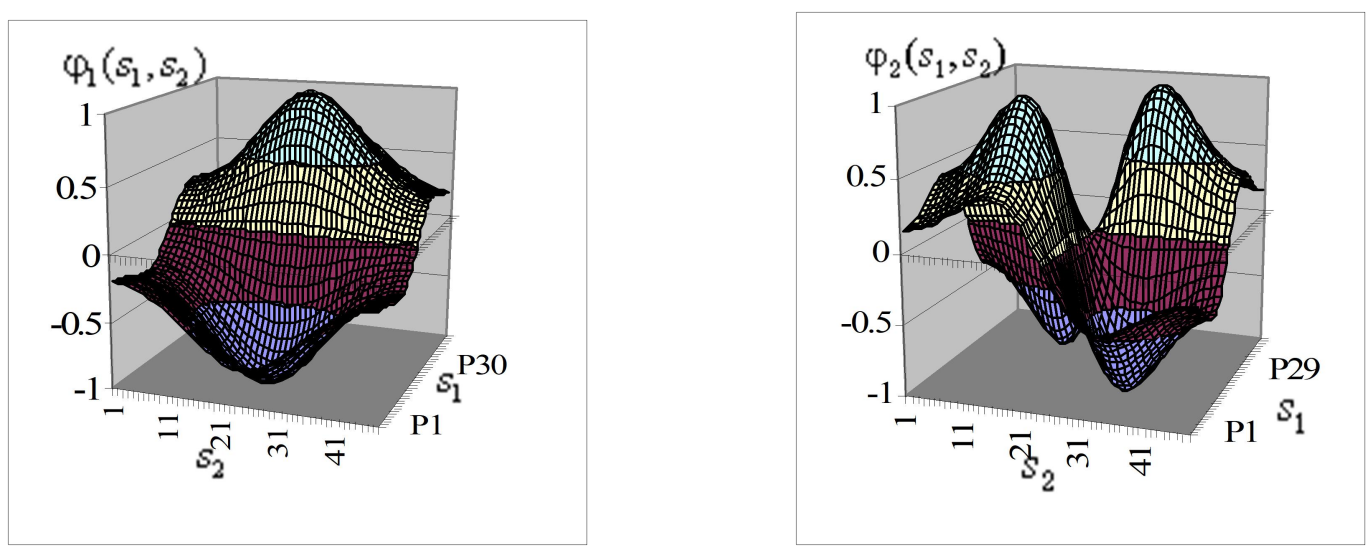

Fig. 3.6. Normalized eigenfunctions of $(3.88)$ corresponding to the eigenvalues: $a-\left(c_{1}=3.43408813477, c_{2}=2.74727050781\right), \quad b-(c 1=$ 4.18890991211, $c 2=4.18890991211)$ 


\subsection{About branching of solutions in the case of a flat aperture}

In $[56,57,74]$ using the found branching lines and eigenfunctions, the analytical investigations of branching of the primary solution of the first type of (3.29) for the case when the the kernel $K\left(s_{1}, s_{2}, s_{1}^{\prime}, s_{2}^{\prime} ; c_{1}, c_{2}\right)$ has the form (3.25), and the multiplicity of eigenvalues of the linear equation (3.37) at the branching points $\left(c_{1}^{(0)}, c_{2}^{(0)}\right)$ is two, are presented.

The study of solutions of (3.29) is realized on the beam $c_{2}=\beta c_{1}$ belonging to the domain $\Lambda_{c}$. Let $\mathbf{c}^{(0)}=\left(c_{1}^{(0)}, c_{2}^{(0)}\right)=\left(c_{1}^{(0)}, \beta c_{1}^{(0)}\right)$ be eigenvalue of (3.37). We assign to parameter $c_{1}^{(l)}$ the small disturbance $c_{1}=c_{1}^{(0)}+\mu, c_{2}=\beta c_{1}^{(0)}+\beta \mu$ and consider the problem on finding all different from $f_{0}\left(s_{1}, s_{2}, c_{1}, c_{2}\right)$ solutions of (3.29), which at $\mu \rightarrow 0$ satisfy the conditions $\max _{Q \in \bar{\Omega}}\left|u\left(Q, c_{1}, c_{2}\right)-f_{0}\left(Q, c_{1}^{(0)}, \beta c_{1}^{(0)}\right)\right| \rightarrow 0, \max _{Q \in \Omega}\left|v\left(Q, c_{1}, c_{2}\right)\right| \rightarrow 0$. The system of (3.29) by means of expanding the integrand functions is reduced to the corresponding system of LyapunovSchmidt equations, similar to (3.35) and (3.36). Desired solutions are found in the form $u\left(Q, c_{1}, c_{2}\right)=f_{0}\left(Q, c_{1}^{(0)}, \beta c_{1}^{(0)}\right)+w(Q, \mu), \quad v\left(Q, c_{1}, c_{2}\right)=\omega(Q, \mu)$. As a result we obtain [74] that at the points $\left(c_{1}^{(0)}, c_{2}^{(0)}\right)=\left(c_{1}^{(0)}, \beta c_{1}^{(0)}\right)$ from the primary solution $f_{0}\left(s_{1}, s_{2}, c_{1}^{(0)}, \beta c_{1}^{(0)}\right)$ branch-off two complex-conjugate solutions having in the first approximation the form

$$
\begin{gathered}
f_{1,2}^{(1)}\left(s_{1}, s_{2}, c_{1}, \beta c_{1}\right)=f_{0}\left(s_{1}, s_{2}, c_{1}^{(0)}, \beta c_{1}^{(0)}\right)+\left(a\left(s_{1}, s_{2}, c_{1}^{(0)}, \beta c_{1}^{(0)}\right)+\alpha_{020}^{(1)}\left(s_{1}, s_{2}, c_{1}^{(0)}, \beta c_{1}^{(0)}\right) h_{1}^{2}\right) \mu \pm \\
\pm i \frac{\varphi_{1}\left(s_{1}, s_{2}, c_{1}^{(0)}, \beta c_{1}^{(0)}\right)}{\left\|\varphi_{1}\left(s_{1}, s_{2}, c_{1}^{(0)}, \beta c_{1}^{(0)}\right)\right\|} h_{1} \mu^{1 / 2}+O\left(\mu^{3 / 2}\right) .
\end{gathered}
$$

The imaginary part being determined by the properties of eigenfunctions $\varphi_{1}\left(s_{1}, s_{2}, c_{1}^{(0)}, \beta c_{1}^{(0)}\right)$. Functions , $\arg f_{1,2}^{(1)}\left(s_{1}, s_{2}, c_{1}, \beta c_{1}\right)$, obtained on the base of (3.57), determine the properties of the phase DP and APD of the field in aperture. The properties obtained in the first approximation of solutions agreewith numerical results.

For example, in Fig. 3.7 are shown the values of the functional $\sigma_{F}$ at $F\left(s_{1}, s_{2}\right) \equiv 1$, which it takes on the primary (curve 1 ) and branching-off (curves 2, 3, 4) solutions on the beam $c_{2}=0.8 c_{1}$. Note, that on the segment $\left(c_{1}^{(1)}, c_{1}^{(5)}\right)$ the branching-off solutions with an odd phase DP $\arg f\left(s_{1}, s_{2}\right)$, to which the nonsymmetric amplitude-phase distribution of the field in aperture corresponds, are the most effective. On the segment $\left(c_{1}^{(5)}, 8\right)$ the most effective is the solution of 4 with properties $\arg f_{1,2}^{(1)}\left(-s_{1}, s_{2}\right)=\arg f_{1,2}^{(1)}\left(s_{1}, s_{2}\right), \arg f_{1,2}^{(1)}\left(s_{1},-s_{2}\right)=\arg f_{1,2}^{(1)}\left(s_{1}, s_{2}\right)$. The 
symmetric but complex APD of the field in aperture corresponds to it. From the analysis of Fig. 3 follows that the same efficiency of the synthesis can be achieved on the branching-off solutions at smaller sizes of aperture and smaller values of parameters $c_{1}, c_{2}$, than on the primary solution. The linear size of aperture can be decreased by the amount $\delta_{1} c_{1}$ or $\delta_{2} c_{1}$ at realization of branching-off solution.

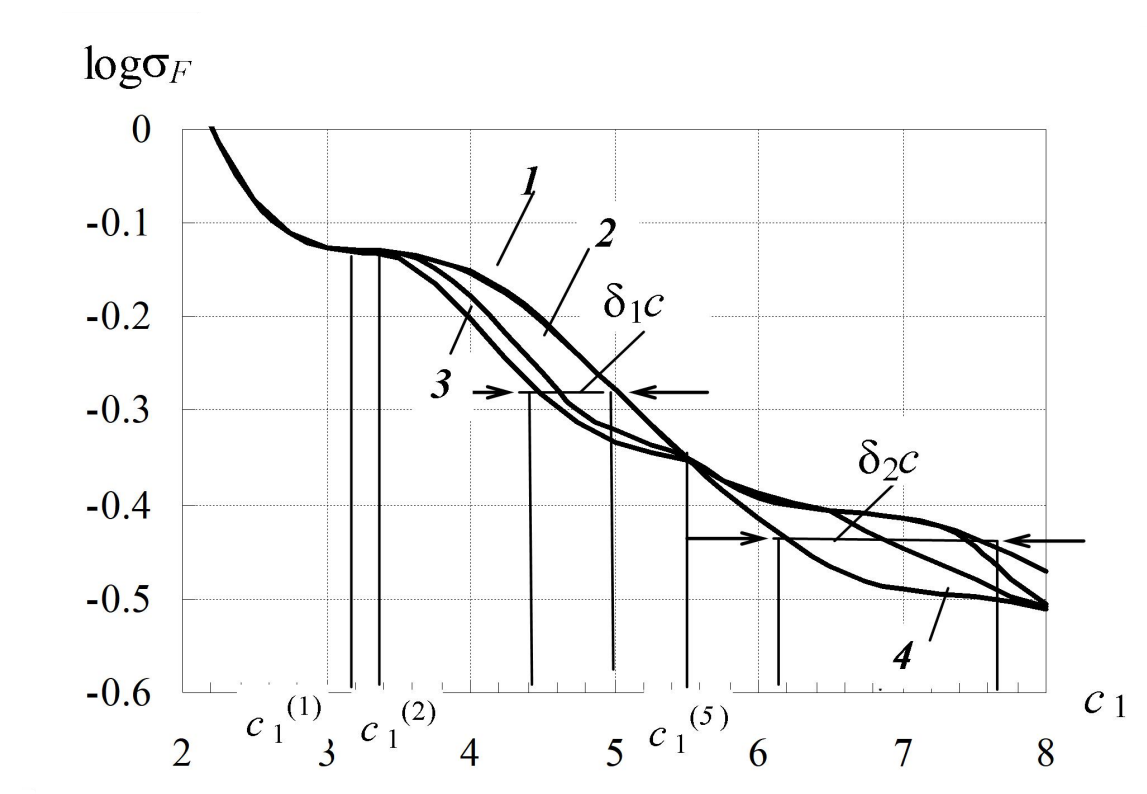

Fig. 3.7

Numerical examples of synthesis of given funnel-shaped amplitude DP defined in the domain $G$ by (3.54), are given in Fig. 3.8 and Fig. 3.9. The branching lines of solutions of (3.27) for this DP are shown in Fig. 3.5. The given DP and optimum synthesized DP are presented in Fig. 3.8, $a$ and Fig. 3.b, respectively, at $c_{1}=9.25, c_{2}=7.4$. The optimum amplitude distributions of the fields in aperture $U(x, y)$, forming the given in Fig. 3.8,b synthesized DP, are shown in Fig. 3.9. From the analysis of these figures we see that the symmetric amplitude DP (Fig. 3.8,b) with graphic accuracy can be created by different distributions of the field in aperture of radiating system, including real and nonsymmetric distribution (Fig. 3.9,b) 


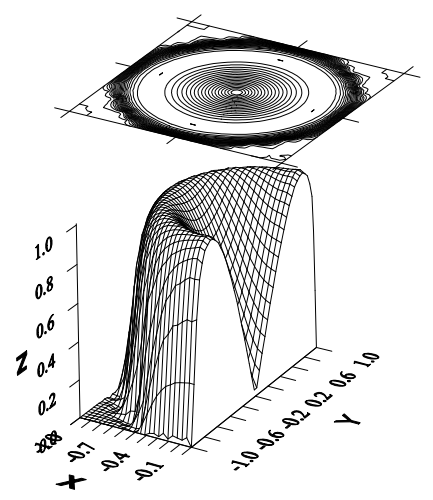

$a$

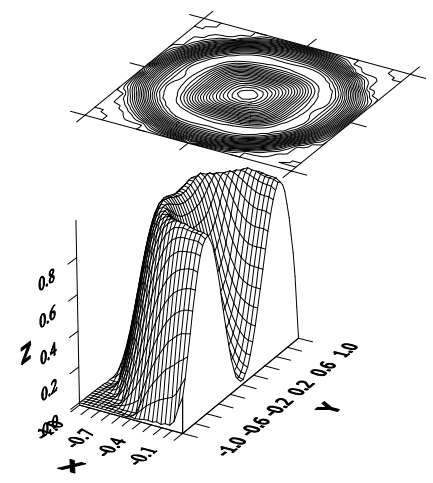

$b$

Fig. 3.8. The prescribed $(a)$ and synthsized $(b)$ DP

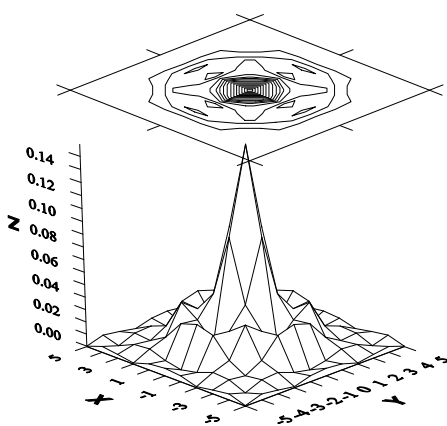

$a$

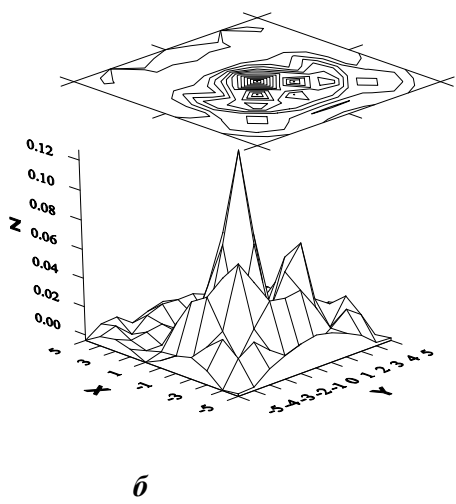

Fig 3.9. Amplitude distributions of currents corresponding to different types of solutions of (3.27)

\section{Synthesis of discrete radiating systems - antenna arrays (AA)}

The investigations of nonlinear synthesis problems of linear and planar antenna arrays (AA) according to the prescribed amplitude DP are presented partially in [7, 24, 28-37]. In the basis of construction of mathematical models it is assumed $[6,27]$ that the excitation of each radiator is characterized by a single complex number $I_{n}$ - complex amplitude of excitation, the physical meaning of which depends on the type of radiating system. Taking into account the linearity of Maxwell's equations, the complex amplitudes of excitation enter linearly in the expression for DP of array, that is

$$
\mathbf{f}(\theta, \varphi)=\sum_{n=1}^{N} I_{n} \mathbf{f}_{n}(\theta, \varphi) e^{i k\left(x_{n} \sin \theta \cos \varphi+y_{n} \sin \theta \sin \varphi+z_{n} \cos \theta\right)} .
$$


Here $\mathbf{f}_{n}(\theta, \varphi)=f_{\theta}^{(n)}(\theta, \varphi) \mathbf{i}_{\theta}+f_{\varphi}^{(n)}(\theta, \varphi) \mathbf{i}_{\varphi}$ is a vector DP of $n$-th radiator. Vector $\mathbf{I}=\left\{I_{1}, I_{2}, \ldots, I_{N}\right\}$ is called the vector of excitation of array or vector of amplitude-phase distribution of currents in the array. Such formulation of DP of array is used in the synthesis problems with regard for mutual influence of radiators $[7,29,30]$. Thus the problem on finding the functions $\mathbf{f}_{n}(\theta, \varphi)$ is reduced to solution of the corresponding boundary problem of electrodynamics in multiply connected domains $[2,4,39,40]$.The method of integral equations [40, 42] is used widely in such classes of problems. The synthesis method of antenna arrays with cylindrical dipoles with account of mutual influence is proposed in [29, 34]. Analysis of nonuniqueness problem of solutions is studied there by means of computational experiments.

In the problems of analysis and synthesis of antenna arrays with many elements is used simplified mathematical model of AA $[5,6]$. It is assumed [6] that AA consists of $N$ identical and identically oriented in space radiators, and vector DP of radiators are identical for all emitters, i.e $\mathbf{f}_{n}(\theta, \varphi)=\mathbf{f}^{(R)}(\theta, \varphi) \quad(n=1 \div N)$. Formula (4.1) for DP of flat AA takes the form

$$
\mathbf{f}(\theta, \varphi)=\mathbf{f}^{(R)}(\theta, \varphi) f_{\Sigma}(\theta, \varphi) \equiv \mathbf{f}^{(R)}(\theta, \varphi) \sum_{n=-M_{1}}^{M_{1}} \sum_{m=1}^{N(n)} I_{n m} e^{i\left(c_{1} x_{n m} s_{1}+c_{2} y_{n m} s_{2}\right)}
$$

Here $s_{1}=(\sin \theta \cos \varphi) / \sin \gamma_{1}, \quad s_{2}=(\sin \theta \sin \varphi) / \sin \gamma_{2}$ are the generalized angular coordinates, $c_{1}=k d_{x} \sin \gamma_{1}, c_{2}=k d_{y} \sin \gamma_{2}$

are dimensionless numerical parameters characterizing the distance between the radiators and the domain (solid angle) $G$, in which the required amplitude DP $F\left(s_{1}, s_{2}\right)$ is given. Since in (4.2) only the second multiplier depends on the vector APD of excitation currents in the array:

$$
f_{\Sigma}(\theta, \varphi)=A \mathbf{I} \equiv \sum_{n=-M_{1}}^{M_{1}} \sum_{m=1}^{N(n)} I_{n m} e^{i\left(c_{1} x_{n m} s_{1}+c_{2} y_{n m} s_{2}\right)},
$$

only the synthesis problem of factor of AA is considerd. Function $f_{\Sigma}\left(s_{1}, s_{2}\right)$ is $2 \pi / c_{1}$ - periodic by argument $s_{1}$ and $2 \pi / c_{2}$ - periodic by $s_{2}$. We consider also (4.3) as the action of the operator $A$ from a finite-dimensional space $H_{I}=\mathbb{C}^{N}$ ( $N$ is number of radiators) into the finite-dimensional subspace of the space, $H_{f}=\mathbf{C}\left(\bar{\Omega}_{P}\right)$ where $\bar{\Omega}_{P}$ is the domain corresponding to the period of array. Let the amplitude DP $F\left(s_{1}, s_{2}\right)$ be given in the domain $\bar{G} \subset \bar{\Omega}_{P}$, and on the set $\bar{\Omega}_{P} \backslash \bar{G}$ is identically equal to zero. The synthesis problem is to minimize the functional [35] 


$$
\min _{\mathbf{I} \in H_{I}} \sigma_{F}(\mathbf{I})=\left\|F-\left|A \mathbf{I}\left\|_{\mathbf{C}_{\left(\overline{\bar{\Omega}_{P}}\right)}^{(2)}}^{2} \equiv\right\| F-\right| f\right\|_{\mathbf{C}_{\left(\overline{\Omega_{P}}\right)}^{(2)}}^{2} .
$$

The basic of synthesis equations of multiplier of AA have the form

$$
\mathbf{I}=A^{*}\left(F \mathrm{e}^{i \arg A \mathbf{I}}\right)
$$

- the equation concerning APD of currents in AA, where $A^{*}$ is conjugate with $A$ operator, and

$$
f(Q)=\mathbf{B} f \equiv \iint_{\bar{G}} K_{\text {ar }}\left(Q, Q^{\prime}, \mathbf{c}\right) F\left(Q^{\prime}\right) \mathrm{e}^{i \arg f\left(Q^{\prime}\right)} d Q^{\prime}
$$

is equation concerning of synthesized DP. Here $Q=\left(s_{1}, s_{2}\right), d Q=d s_{1} d s_{2}, \quad \mathbf{c}=\left(c_{1}, c_{2}\right)$; $K_{\mathrm{ar}}\left(Q, Q^{\prime}, \mathbf{c}\right)$ is the kernel the form of which depends on the distribution of elements in AA. In particular, in the case of a rectangular array with number of elements $N_{1} \cdot N_{2}=\left(2 M_{1}+1\right) \cdot\left(2 M_{2}+1\right)$ the kernel $K_{\text {ar }}\left(Q, Q^{\prime}, \mathbf{c}\right)$ is writtern as

$$
K_{\mathrm{ar}}\left(Q, Q^{\prime}, c_{1}, c_{2}\right)=\frac{\sin \left(N_{1} \frac{c_{1}}{2}\left(s_{1}-s_{1}^{\prime}\right)\right)}{\sin \left(\frac{c_{1}}{2}\left(s_{1}-s_{1}^{\prime}\right)\right)} \cdot \frac{\sin \left(N_{2} \frac{c_{2}}{2}\left(s_{2}-s_{2}^{\prime}\right)\right)}{\sin \left(\frac{c_{2}}{2}\left(s_{2}-s_{2}^{\prime}\right)\right)} .
$$

To find the possible branching lines of solutions of (4.6) a linear homogeneous integral equation

$$
\varphi(Q)=T\left(c_{1}, c_{2}\right) \varphi \equiv \iint_{\bar{G}} F\left(Q^{\prime}\right) K_{\mathrm{ar}}\left(Q, Q^{\prime}, c_{1}, c_{2}\right) / f_{0}\left(Q^{\prime}, c_{1}, c_{2}\right) \varphi\left(Q^{\prime}\right) d Q^{\prime},
$$

is obtained where $f_{0}\left(Q^{\prime}, c_{1}, c_{2}\right)$ is a primary solution of (4.6).

Note that the kernel $K_{\text {ar }}\left(Q, Q^{\prime}, c_{1}, c_{2}\right)$ is degenerate. Consequently, Equation (4.8) is reduced to the corresponding homogeneous SLAE what in a special case of rectangular array has the form

$$
x_{k l}=\sum_{m=-M_{1}}^{M_{1}} \sum_{n=-M_{2}}^{M_{2}} a_{n m}^{(k l)}\left(c_{1}, c_{2}\right) x_{n m} \quad\left(k=-M_{1} \div M_{1}, l=-M_{2} \div M_{2}\right)
$$

Coefficients of this system depend nonlinearly on the spectral parameters $c_{1}, c_{2}$ and on the given amplitude DP. In $[35,65]$ the conditions are determined and the existence theorem of connected components of the spectrum of (4.8) is proved. To find the spectral lines the implicit function method (3.46) and (3.47), is used.

Consider the numerical results of finding the solutions of the branching lines in the synthesis problems of a plane equidistant antenna array with $11 \times 11$ radiators for two given in 
the domain $\bar{G}=\left\{\left(s_{1}, s_{2}\right):\left|s_{1}\right| \leq 1,\left|s_{2}\right| \leq 1\right\} \quad$ amplitude DPs $F\left(s_{1}, s_{2}\right)=\cos \left(\pi s_{1} / 2\right)\left|\sin \left(\pi s_{2}\right)\right|$

(Fig.4.2)

and $F\left(s_{1}, s_{2}\right)=\left|\sin \left(\pi s_{1}\right)\right| \cdot\left|\sin \left(\pi s_{2}\right)\right|($ Fig. 4.2), which are obtained by solving of (4.8) and (4.9). The prescribed and synthesized amplitude DPs (with phase DP odd by argument $s_{2}$ ) at $c_{1}=1.25$, $c_{2}=1.125$, are shown in Fig 4.3 and Fig. 4.4, respectively. The amplitude and phase distributions of currents in the array of corresponding synthesized DP are given in Fig. 4.5. From the analysis of this figure we see that nonsymmetric $Y$ - direction distribution of currents in the array forms symmetrical amplitude DP.

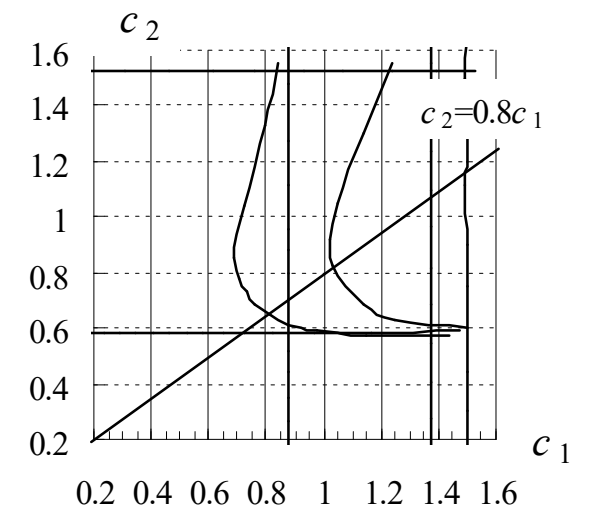

Fig. 4.1. The possible branching lines of solutions of (4.6) for $F\left(s_{1}, s_{2}\right)=\cos \left(\pi s_{1} / 2\right)\left|\sin \left(\pi s_{2}\right)\right|$

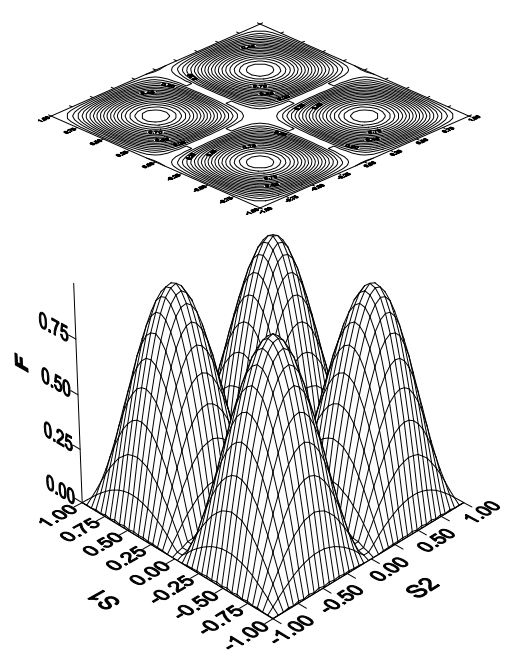

Fig. 4.3. The prescribed DP

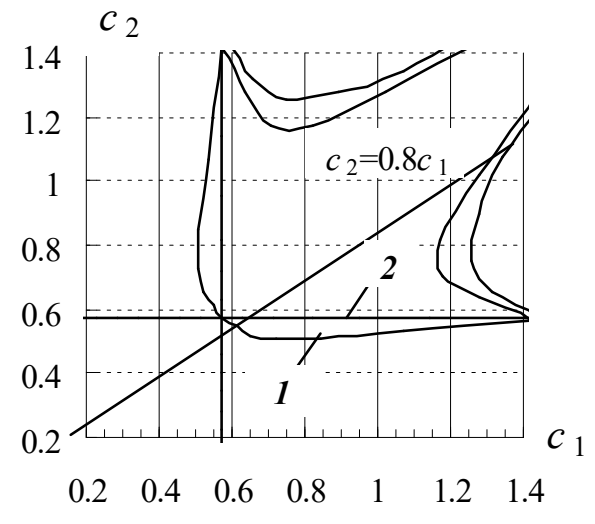

Fig. 4.2. The possible branching lines of solutions of (4.6) for $F\left(s_{1}, s_{2}\right)=\left|\sin \left(\pi s_{1}\right)\right| \cdot\left|\sin \left(\pi s_{2}\right)\right|$
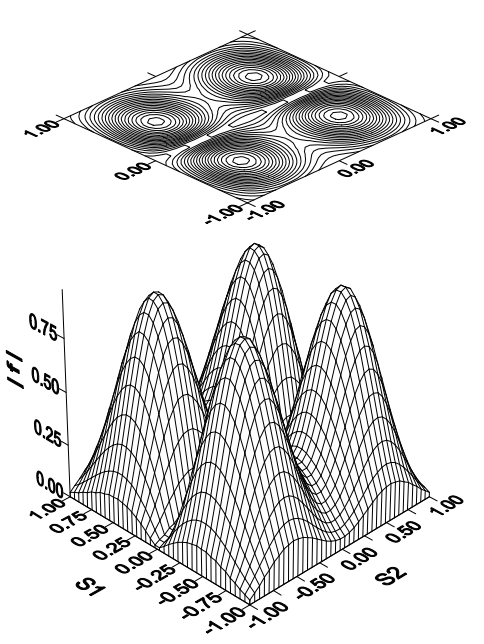

Fig. 4.4. The synthesized DP 

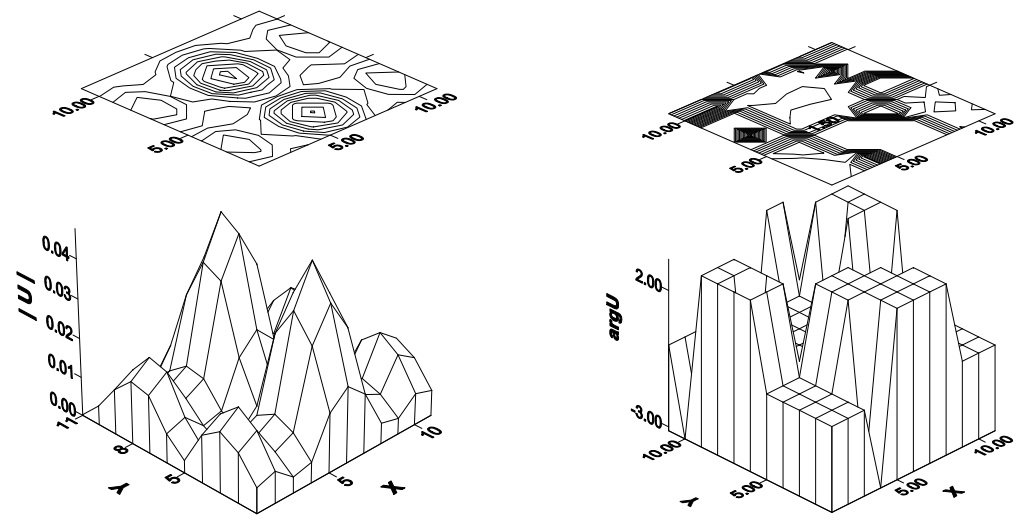

$\boldsymbol{a}$

b

Fig. 4.5. The optimal amplitude (a) and phase (b) distributions of currents in the array

\section{Numerical methods of solution of the basic synthesis equations}

The above results show that the nonlinear synthesis problems according to the prescribed amplitude DP and given energy DP have nonunique solutions. Application of the methods of branching theory of solutions to nonlinear integral equations allows to determine the quantity of existing solutions, to find solutions in the first approximation and to determine their quality characteristics. To find the complete solutions of these equations numerical methods $[7,29,36,49,75]$ are applied. The defined properties of solutions obtained by analytical investigations make it possible to choose the initial approximation having the basic properties of the desired solutions and they are placed in certain neighborhoods of complete solutions.

Conditionally the process of numerical solution of synthesis problem can be divided into two stages. The first of them is described partially above and it consists in finding the points (lines) of branching and determination of types of existings solutions depending on the value of physical parameters. The second stage consists in solving the basic synthesis equations by iterative methods.

\subsection{Numerical solution of synthesis equations corresponding to functional $\sigma_{F}$}

As an example of the scalar problem we consider iterative process of solving the eqution of type (2.9), in the base of which we put the successive approximations method $[7,75]$

$$
f_{n}=A A^{*}\left(F e^{i \arg f_{n-1}}\right) \quad(n=1,2,3, \ldots) .
$$


Obviously, the successive approximations method (5.1) is equivalent to the following iterative process

$$
\left\{\begin{array}{l}
U_{n}=A^{*}\left(F e^{i \arg f_{n-1}}\right), \\
f_{n}=A U_{n}
\end{array} \quad(n=1,2,3, \ldots) .\right.
$$

In [75] it is shown that the sequences $\left\{U_{n}\right\}$ and $\left\{f_{n}\right\}$ generated by iterative process (5.2), are relaxational for functional $\sigma_{F}$. Relaxation properties of (5.2) states

Theorem 5.1. The sequence $\left\{U_{n}\right\}$ is generated by the iterative process (5.2), it is relaxation for functional $\sigma_{F}(U)$, and the values which it takes on $\left\{U_{n}\right\}$ form a convergent numerical sequence $\left\{\sigma_{F}\left(U_{n}\right)\right\}$.

Formulate also the properties of the operator $B$ entering in (3.5) that complement the properties of $1^{\circ}-3^{\circ}$ solutions of (3.5), presented in Sec. 3.1.

Theorem 5.2. Nonlinear operator $B$, defined by (3.5), acts in the space $\mathbf{C}[-1,1]$ of continuous complex-valued functions, it is a compact and maps set $S_{M}=\left\{f:\|f\|_{C} \leq M\right\}$ it into itself, where

$$
M=\max _{s \in[-1,1]} \int_{-1}^{1} F\left(s^{\prime}\right)\left|K\left(s, s^{\prime} ; c\right)\right| d s^{\prime},
$$

that is $B\left(S_{M}\right) \subset S_{M}$.

From the proved theorem follows, in particular, the following fact. Since the solutions of (3.5) are fixed points of the operator $B$, from the relation $B\left(S_{M}\right) \subset S_{M}$ follows that all solutions of this equation belong to the set $B\left(S_{M}\right) \subset S_{M}$. In addition, is valid [75]

Corollary 5.1. If the sequence $\left\{f_{n}\right\}$ which is generated by the iterative process (5.2), is minimizing for the functional $\sigma_{F}(U)$, then from $\left\{f_{n}\right\}$ can be selected a subsequence $\left\{f_{n_{k}}\right\}$ converging uniformly to the minimum point $f_{*}$ of the functional $\sigma_{F}(U)$.

Note that theorem 5.2 and corollary 5.1 are extended to the case of synthesis problem of a flat aperture with use of equation of the type (3.27).

\subsection{Numerical solution of synthesis equations corresponding to functional $\sigma_{F_{\alpha}}$}

5.2.1. In the base of construction of iterative processes of solving the nonlinear operator equations of the type (2.12) and (2.13) we put implicit scheme of the successive 
approximations method $[7,76]$. In a general case, the iterative process of solution of $(2.12)$ has the form

$$
\left(E+\alpha^{-1} A^{*} A\right) U_{n+1}=\alpha^{-1} A^{*}\left(F \exp \left(i \arg \left(A U_{n}\right)\right)\right) \quad(n=0,1,2, \ldots),
$$

where $E$ is an identity operator acting in the space $H_{U}=L_{2}(V)$.

The implicit scheme of iteration process for (2.13) with respect to synthesized DP $f^{3}$ has the form similar to $(5.4)$

$$
\left(E+\alpha^{-1} A A^{*}\right) f_{n+1}=\alpha^{-1} A A^{*}\left(F \exp \left(i \arg \left(f_{n}\right)\right)\right) \quad(n=0,1,2, \ldots) .
$$

Note that the implicit schemes (5.4) and (5.5) are characterized by the fact that linear operator equation is solved on every iteration step. In addition the question of solvability of (5.4) and (5.5) appears, to which a positive answer gives a theorem about the solvability of the functional equation of the second kind of the type

$$
x=W x+y
$$

in the Banach space $X$, where $W$ is a linear compact operator [69].

Theorem 5.3. In order that (5.6) have the solution at an arbitrary $y \in X$, it is necessary and sufficiently that homogeneous equation $x=W x$ have a unique solution (obviously, that $x=0)$.

For a sequence $\left\{U_{n}\right\}$ obtained by (5.4), is valid

Theorem. 5.4. Let $A: L_{2}(\bar{V}) \rightarrow L_{2}(\bar{G})$ be a completely continuous operator, $F$ be a continuous real nonnegative function in $\bar{G}$ and at $0<\alpha<\infty$ there exists the inverse operator $\left(E+\alpha^{-1} A^{*} A\right)^{-1}$, in addition, the dimension of the space of zeros $N(A)=0$.

Then the sequence $\left\{U_{n}\right\}$ generated by the iterative process (5.4), is a minimizing for the functional

$$
\left\|\operatorname{grad} \sigma_{F_{\alpha}}\left(U_{n}\right)\right\|_{H_{I}}=\left\|A^{*} F\left(\exp \left(i \arg A U_{n}\right)\right)-A^{*} A U_{n}-\alpha U_{n}\right\|_{H_{U}}
$$

in the space $H_{I}$.

\footnotetext{
${ }^{3}$ Comment 5.1. Since the set of continuous functions in the domain $G$ is adense everywhere in the space $L_{2}(G)$, and completion of space $C_{(G)}^{(2)}$ of continuous functions as the normalized space (with norm $\|x\|_{C_{(G)}^{(2)}}=\sqrt{(x, x)}$ ), coincides with the Hilbertian space $L_{2}(G)$ [14], justification of the convergence of (5.5) concerning (2.13) can be considered in the Hilbertian space $H_{f}=L_{2}(G)\left(\bar{G} \subset \mathbb{R}^{2}\right.$ or $\left.\bar{G} \subset \mathbb{R}^{1}\right)$.
} 
We denote the operator in right part of (5.4) as:

$$
D(U)=\alpha^{-1}\left(E+\alpha^{-1} A^{*} A\right)^{-1} A^{*}(F \exp (i \arg (A U)) .
$$

For the operator $D(U)$ is valid

Lemma 5.1. Let $A: H_{U} \rightarrow H_{f}$ be a completely continuous operator. Then the operator $D(U)$ defined by (5.8), is compact and it transfers any bounded set $U_{r}=\left\{U:\|U\|_{H_{U}} \leq r\right\}$ into its relatively compact part at $\alpha^{-1}\left\|A^{*}\right\|\|F\|_{H_{f}} \leq r<\infty$.

Depending on the choice of initial approach the successive approximations (5.4) can converge to the solutions of various types [80]. Thus, it is shown that there is true

Corollary 3.2. If $\operatorname{grad} \sigma_{\alpha}(U)$ is operator continuous in some neighborhood $U_{*} \subset H_{U}$ of the point $U_{*}$, then from Theorem 5.2 and Lemma 5.1 follows that the subsequence $\left\{U_{n_{k}}\right\}$ converges to some solution of (2.12) by the norm of the space $H_{U}$ if $U_{0} \in U_{*}$.

\subsection{Numerical solution of synthesis problems with use of the energy criterion $\sigma_{F}$}

First we shall consider the iterative process of solution the equation of type (2.34) in the Hilbertian space $H_{U}=L_{2}(V) \otimes L_{2}(V) \otimes L_{2}(V)$ under certain restrictions on the parameter $\alpha$. This equation is written as

$$
\left(E-\frac{2}{\alpha} A^{*} N_{0} A\right) \mathbf{U}=-\frac{2}{\alpha} A^{*}\left(|A \mathbf{U}|^{2} \cdot A \mathbf{U}\right)
$$

where $E: H_{U} \rightarrow H_{U}$ is an identity operator, $A: H_{U} \rightarrow \mathbf{C}(\bar{G})$ is completely continuous operator. We denote $\|A\|_{H_{U} \rightarrow \mathbf{C}(G)}=\beta$. Note that the scalar product and the corresponding norm $\|\cdot\|_{H_{U}}$ in the space $H_{U}$ are defined by (3.56) and (3.57), and the Chebyshev $\|\cdot\|_{\mathbf{C}(G)}$ and mean-square $\|\cdot\|_{\mathbf{C}_{(\bar{G}}^{(2)}}$ norms in the space $\mathbf{C}_{(\bar{G})}^{(2)}$ are introduced by formulas (3.56), (3.57) and (3.58).

Henceforth we shall consider completion of the space $\mathbf{C}_{(\bar{G})}^{(2)}$ relatively to the norm $\|\cdot\|_{\mathbf{C}_{(G)}^{(2)}}$ [14], which is the Banach space and coincides with the Hilbertian space $H_{f}=L_{2}(\bar{G}) \otimes L_{2}(\bar{G})$, the norm in which we shall denote by symbol $\|\cdot\|_{L_{2}(\bar{G})}$. We assume that $A: H_{U} \rightarrow H_{f}$ is a completely continuous operator and in the space $\mathbf{C}_{(\bar{G})}^{(2)}$ the domain of its values $R(A)$ is a set of continuous functions. 
Taking into account the equality $A \mathbf{U}=\mathbf{f}$ we shall consider the expression $N_{0} A \mathbf{U}$ in (5.9) as an operator of multiplication by the function $N_{0}$ :

$$
\mathbf{N}(\mathbf{f})=N_{0} \cdot \mathbf{f},
$$

acting in the space $H_{f}$ where $N_{0}$ is real nonnegative continuous function on the compact $\bar{G}$, in addition $\left\|N_{0}\right\|_{\mathbf{C}(G)}=1$. Obviously, that (5.10) is a linear bounded operator, and $\|\mathbf{N}\|_{H_{f} \rightarrow H_{f}} \leq 1$.

If $\alpha>2\left\|A^{*} \mathbf{N} A\right\|$, then there exists the inverse operator $\left(E-\frac{2}{\alpha} A^{*} \mathbf{N} A\right)^{-1}$, the norm of which satisfies the inequality [14]

$$
\left\|\left(E-\frac{2}{\alpha} A^{*} \mathbf{N} A\right)^{-1}\right\| \leq \frac{1}{1-\frac{2}{\alpha}\left\|A^{*} \mathbf{N} A\right\|} .
$$

In this case, Equation (5.9) we shall write as

$$
\mathbf{U}=D(\mathbf{U}) \equiv-\frac{2}{\alpha}\left(E-\frac{2}{\alpha} A^{*} \mathbf{N} \mathbf{U}\right)^{-1} A^{*}\left(|A \mathbf{U}|^{2} \cdot A \mathbf{U}\right) .
$$

Here we shall show that the solution of (5.12) can be obtained as a limit of successive approximations of the iterative process [61]:

$$
\mathbf{U}_{n+1}=t \mathbf{U}_{n}+(1-t) D\left(\mathbf{U}_{n}\right) \quad(n=0,1,2, \ldots),
$$

where $t$ is some fixed number with the interval $(0,1)$. In addition successive approximations can converges to different solutions of (5.12) depending on the choice of the initial approximation.

To determine the conditions and to justify convergence of (5.13), we shall use the Theorem 4.1 with $[61$, p. 68], according to which: if nonexpanding operator $W$ converts a closed convex set $\omega$ of strictly convex Banach space $X$ into its compact part, then successive approximations

$$
x_{n+1}=t x_{n}+(1-t) W\left(x_{n}\right) \quad(n=0,1,2, \ldots),
$$

where $t$ is any fixed number from the interval $(0,1)$, converges to some solution of the equation $x=U(x)$ at some $x_{0} \in \omega$.

Since the Hilbertian space $H_{U}$ is strictly convex Banach space (see [61, p. 67]), then to satisfy of the conditions of this theorem concerning (5.12), it is sufficiently to show that a 
closed convex set $S_{r_{0}}$ exists in the space $H_{U}$, where the operator $D(\mathbf{U})$ is nonexpanding and completely continuous. In addition there is such relation $D\left(S_{r_{0}}\right) \subset S_{r_{0}}$.

Satisfication of these conditions results from lemmas, proved in [7, 49].

Lemma 5.2. Let $A: H_{U} \rightarrow H_{f}$ be a linear completely continuous operator and the domain of its values $R(A)$ is a set of continuous functions, $\alpha>2\left\|A^{*} N_{0} A\right\|$. Then $D(U)$ is a nonexpanding operator on $S_{r_{0}} \subset L_{2}(\bar{V})$, where

$$
S_{r_{0}}=\left\{\mathbf{U}:\|\mathbf{U}\|_{H_{U}} \leq r_{0}\right\}, \quad r_{0}=\left(\frac{1-\mu\left\|A^{*} N_{0} A\right\|}{3 \mu \beta^{3}\left\|A^{*}\right\|}\right)^{1 / 2}, \quad \mu=2 / \alpha,
$$

that is, for any $\mathbf{U}_{1}, \mathbf{U}_{2} \in S_{r_{0}}$ the inequality

$$
\left\|D\left(\mathbf{U}_{1}\right)-D\left(\mathbf{U}_{2}\right)\right\|_{H_{U}} \leq\left\|\mathbf{U}_{1}-\mathbf{U}_{2}\right\|_{H_{U}}
$$

is satisfied.

Lemma 5.3. Let $A: H_{U} \rightarrow H_{f}$ be a linear completely continuous operator and the domain of its values $R(A)$ is a set of continuous functions, $\alpha>2\left\|A^{*} \mathbf{N} A\right\|$. Then $D(\mathbf{U}): H_{U} \rightarrow H_{U}$, defined by (5.12), is a completely continuous operator for which the relation $D\left(S_{r_{0}}\right) \subset S_{r_{0}}\left(S_{r_{0}}\right.$ is a closed convex set, defined by (5.14)), is satisfied.

\subsection{Numerical solution of synthesis problems with optimization of geometry of radiating system}

In this section we shall consider the synthesis problem of a flat aperture according to the prescribed amplitude DP for the case when the form of aperture and amplitude-phase distribution of the field (currents) in it is optimized simultaneously, limiting by the case of linear polarization[25, 26, 77]. We shall consider a special case when the field in the aperture is linearly polarized along one of the coordinate axes, and DP has only one component. We introduce inside of aperture the polar coordinate system $x=r \cos \psi, y=r \sin \psi$. Let $\rho(\psi)$ be a function of the boundary of aperture $\bar{S}$. Then DP $f\left(s_{1}, s_{2}\right)$ which is formed by amplitudephase distribution of the field in the aperture $U(r, \psi)$, is given by the formula $[7,16]$

$$
f_{g_{j}}\left(s_{1}, s_{2}\right)=A_{j}\left(U_{v}, \rho\right) \equiv \int_{0}^{2 \pi \rho(\psi)} \int_{0} U_{v}(r, \psi) e^{i k r\left(s_{1} \cos \psi+s_{2} \sin \psi\right)} r d r d \psi \quad(j=1, v=x ; j=2, v=y) .
$$


Later on we omit the index in definition of $f$. Let the given amplitude DP $F\left(s_{1}, s_{2}\right)$ be different from identical zero in some limited closed domain $\bar{G} \subset \Omega=\mathbb{R}^{2}$ and it is identically equal to zero at $\left(s_{1}, s_{2}\right) \in \Omega \backslash \bar{G}$. The problem of simultaneous synthesis of the aperture shape $S$ and amplitude-phase distribution of the field in it is considered as the problem on finding the functions $U(r, \psi)$ and $\rho(\psi)$ minimizing the functional

$$
\tilde{\sigma}_{F}(U, \rho)=\iint_{G}\left[F\left(s_{1}, s_{2}\right)-\left|f\left(s_{1}, s_{2}\right)\right|\right]^{2} d s_{1} d s_{2}+\iint_{\Omega \backslash G} \mid f\left(s_{1},\left.s_{2)}\right|^{2} d s_{1} d s_{2}+\gamma \int_{0}^{2 \pi \rho(\psi)} \int_{0}^{\rho)} r d r d \psi,\right.
$$

in which the first two summands describe the mean-square deviation of modules of given and synthesized DP's in space $\mathbb{R}^{2}$, and the third one - imposes restrictions on the square of aperture $S$. We shall consider the parameter $\gamma>0$ as a weight coefficient.

We introduce into consideration the following functional spaces: $H_{U}=L_{2}(S)$ is a space of square integrable complex functions in the domain $S, H_{\rho}=L_{2}[0,2 \pi]$ is a space of square integrable real functions on the segment $[0,2 \pi], H_{f}=L_{2}(\Omega)$ is a space of square integrable complex functions in the domain $\Omega$. Scalar products and generated by it norms we shall introduce as follows:

$$
\begin{array}{ll}
\left(U_{1}, U_{2}\right)_{H_{U}}=\int_{0}^{2 \pi \rho(\psi)} \int_{0}^{\rho} U_{1}(r, \psi) \overline{U_{2}(r, \psi) r} d r d \psi, & \|U\|_{H_{U}}=(U, U)^{1 / 2}, \\
\left(\rho_{1}, \rho_{2}\right)_{H_{\rho}}=(1 / 2) \int_{0}^{2 \pi} \rho_{1}(\psi) \rho_{2}(\psi) d \psi, & \|\rho\|_{H_{\rho}}=(\rho, \rho)^{1 / 2}, \\
\left(f_{1}, f_{2}\right)_{H_{f}}=\iint_{\Omega} f_{1}\left(s_{1}, s_{2}\right) \overline{f_{2}\left(s_{1}, s_{2}\right)} d s_{1} d s_{2}, & \|f\|_{H_{f}}=\left(f_{1}, f_{2}\right)^{1 / 2} .
\end{array}
$$

Taking into account the introduced norms, the last summand in (5.17) and Parseval's equality have the form

$$
\gamma \int_{0}^{2 \pi} \int_{0}^{\rho(\psi)} r d r d \psi=\frac{\gamma}{2} \int_{0}^{2 \pi} \rho^{2}(\psi) d \psi=\gamma\|\rho\|_{H_{\rho}}^{2}, \quad\|f\|_{H_{f}}^{2}=(2 \pi / k)^{2}\|U\|_{H_{U}}^{2} .
$$

On this base the functional $\tilde{\sigma}_{F}$ is presented as:

$$
\tilde{\sigma}_{F}(U, \rho)=\|F\|_{H_{f}}^{2}-2(F,|f|)+(2 \pi / k)^{2}\|U\|_{H_{U}}^{2}+\gamma\|\rho\|_{H_{\rho}}^{2} .
$$

We shall consider the iterative process of numerical minimization of (5.19). In it base we shall put the ideas similar, as at minimization of functions of two variables by a 
coordinate descent method. Let $V^{*}=\left(U^{*}, \rho^{*}\right)$ be a minimum point of the functional $\tilde{\sigma}_{F}(U, \rho)$ and $V^{(0)}=\left(U^{(0)}, \rho^{(0)}\right)$ be an initial approximation chosen from some neighborhood of the point $V^{*}$. We shall denote by $S^{(0)}$ the initial shape of aperture, that is described by the function $\rho^{(0)}(\psi)$. Substitute $\rho^{(0)}(\psi)$ in $(5.19)$ and consider its restriction in the space $H_{U}$ :

$$
\tilde{\sigma}_{U}(U)=\tilde{\sigma}_{F}\left(U, \rho^{(0)}\right)
$$

From the necessary condition of the functional minimum $\tilde{\sigma}_{U}(U)$ we obtain equation of type (2.9). Numerically we solve it by successive approximations method, given in pt. 5.1:

$$
f_{n+1}\left(Q^{\prime}\right)=(k / 2 \pi)^{2} \iint_{\bar{G}} F(Q) K\left(Q^{\prime}, Q ; k\right) e^{i \arg f_{n}(Q)} d Q \quad(n=0,1,2, \ldots) .
$$

As a result, we find the function $f^{(1)}(Q)$, and obtain the first approximation of the solution $U^{(1)}$ by the formula of type (2.10).

We shall pass to finding the function $\rho(\psi)$ that describes the boundary of aperture $\bar{S}$. We fix the function $U^{(1)}$ extending its analytically according to (2.10) to the plane $X O Y$ in (5.19), and consider the functional $\tilde{\sigma}_{\rho}(\rho)=\tilde{\sigma}_{F}\left(U^{(1)}, \rho\right)$ which depends only on the function $\rho$. With the necessary minimum condition: $\left(\operatorname{grad} \tilde{\sigma}_{\rho}(\rho), g\right)=\left(\tilde{\sigma}_{\rho}^{\prime}(\rho), g\right)=0$ where $\left(\tilde{\sigma}_{\rho}^{\prime}(\rho), g\right)=0$ is an arbitrary element of the space $H_{\rho}$, we obtain the equation

$$
B(\rho) \equiv \rho(\psi)\left\{\left|\left(\frac{k}{2 \pi}\right)^{2} \iint_{\bar{G}} F(Q) e^{i \arg f(Q)} e^{-i k \rho(\psi) \alpha(Q, \psi)} d Q\right|^{2}-\gamma\left(\frac{k}{2 \pi}\right)^{2}\right\}=0
$$

which is a nonlinear functional equation with respect to the function $\rho(\psi)$.

We shall find numerically solutions of (5.22), using the Newton-Kantorovich method [69]:

$$
\begin{aligned}
& B^{\prime}\left(\rho_{n}\right) \Delta \rho_{n}=-B\left(\rho_{n}\right), \\
& \rho_{n+1}(\psi)=\rho_{n}(\psi)+\Delta \rho_{n}(\psi) \quad(n=0,1,2, \ldots),
\end{aligned}
$$

where $B^{\prime}\left(\rho_{n}\right)$ is the partial Frechet derivates of operator $B$ by the function $\rho$. We assume that $\rho_{0}=\rho^{(0)}$. Equation (5.23) is a linear integral equation of the form

$$
L\left(\rho_{n}(\psi)\right) \Delta \rho_{n}(\psi)+\int_{0}^{2 \pi} M\left[\rho_{n}(\psi), \rho_{n}\left(\psi^{\prime}\right), \psi, \psi^{\prime}\right] \Delta \rho_{n}\left(\psi^{\prime}\right) d \psi^{\prime}=-B\left(\rho_{n}(\psi)\right)
$$


which $L\left(\rho_{n}(\psi)\right) \neq 0$ can be reduced to the Fredholm equation of the second kind at $L\left(\rho_{n}(\psi)\right) \neq 0$. Solving (5.25) we find the first approximation for the function $\rho^{(1)}$ that describes a boundary of aperture of the radiating system.

Continuing finding in turn the approximations of functions $U^{(n)}$ and $\rho^{(n)}$, we obtain the sequence $\left\{U^{(n)}, \rho^{(n)}\right\}$ that is relaxational for (5.17). In more detail the problem of choice of initial approximations and justification of relaxation for functional $\tilde{\sigma}_{F}(U, \rho)$ is given in [].

First we shall consider the numerical results of synthesis of flat aperture with optimization of its geometry. In Fig. 5.1. and Fig. 5.2 the examples of synthesis of amplitude DPs, which in cross section have quasi-square and quasi-triangular shapes, are given. The optimal shapes of apertures are given there too.

Note that the problems of such class arise, in particular, at the synthesis of contour DPs of fixed and variable forms for satellite antenna systems needed for uniform irradiation of a given territorial zone from the board of artificial satellite, where multi-beam antennas are used often [77-82].

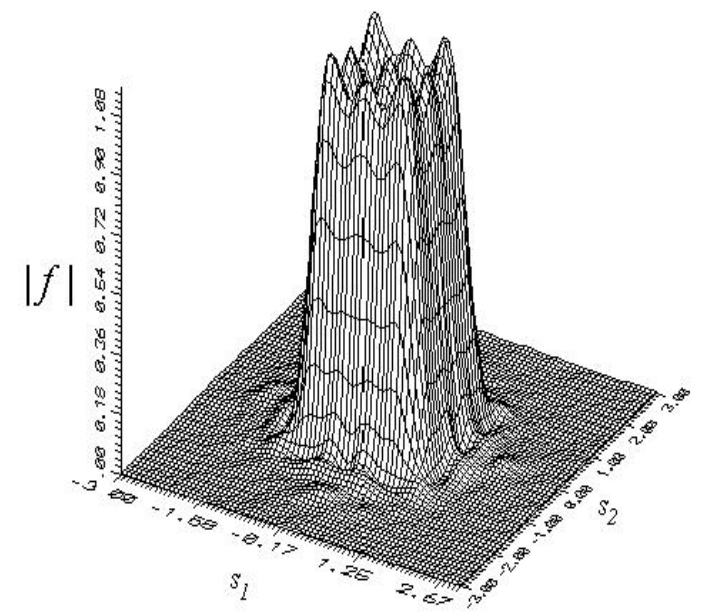

$\boldsymbol{a}$

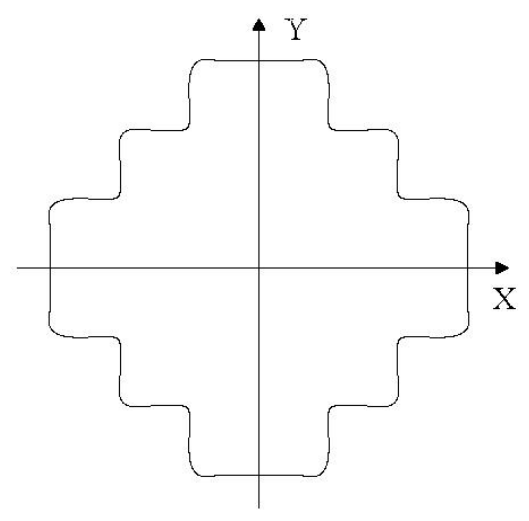

$b$

Fig. 5.1. Synthesis DP with square contour: $a$ - synthesized DP with square contour, $b$ - the optimal shape of aperture 

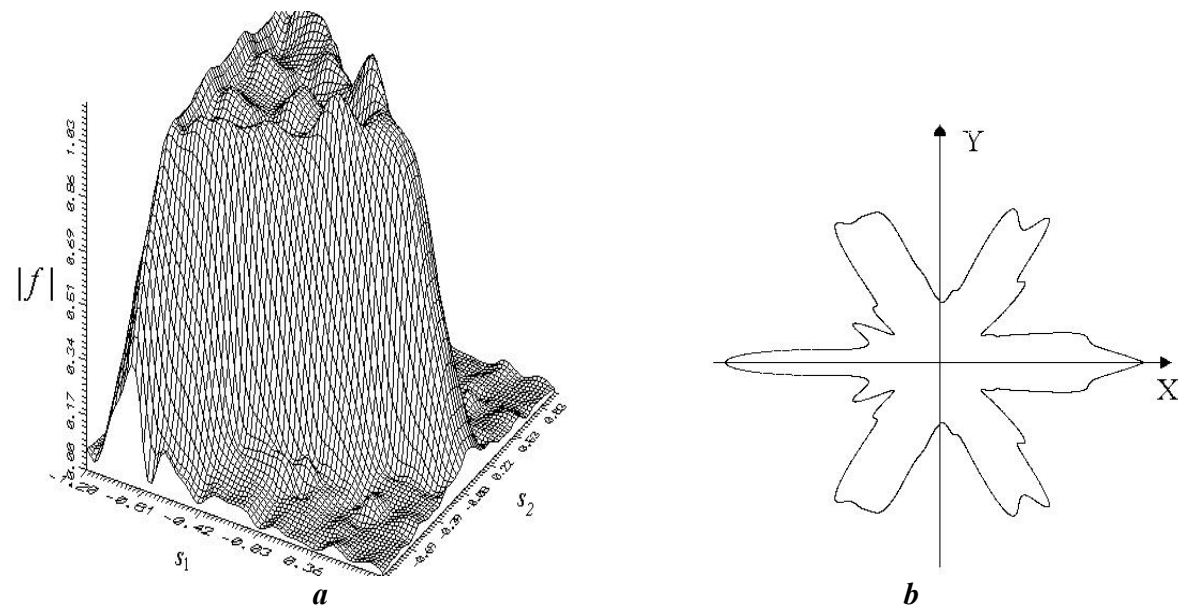

Fig. 5.2. Synthesis of DP with triangular contour: $a-$ synthesized DP with quasi-triangular contour, $b-$ optimal shape of aperture

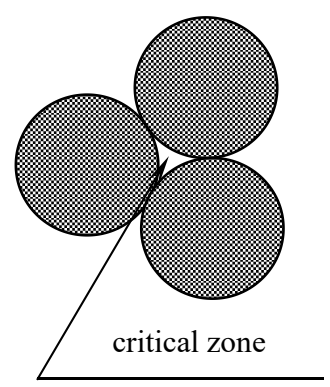

Fig. 5.3

If multibeam antenna (MBA) has a radiating aperture of circular shape, and partial beams in the cross section have the shape of a circle and nonuniform distribution of radiated energy inside of the section, then on the junction of three neighboring rays with a circular cross section the so-called critical zones (Fig. 5.3) with low level of radiated energy occur. One of the possible ways of solution of this problem is passage to alternative forms of apertures that on the base of the optimal APD will form rays that have rectangular, triangular or hexagonal shapes and close to constant (inside of contour) coefficient of directed action in rectangular cross section. Obviously that on the base of such partial beams it is easy to synthesize given summary DP without critical zones. Below the results of synthesis of triangle-beam contour DP with partial beams with circular (Fig. 5.4,a) and quasi-rectangular (Fig. 5.4,b) contours are presented. From the analysis of the figures we see that in the summary DP which is 
obtained on the base of quasi-square of contours, critical zones are absent, and variation of radiated energy inside of the contour does not exceed $2 \mathrm{~dB}$.

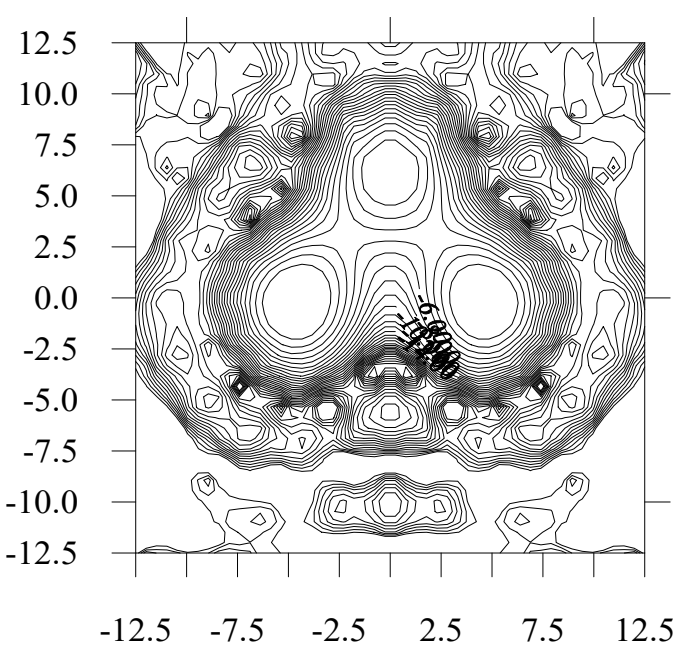

$\boldsymbol{a}$

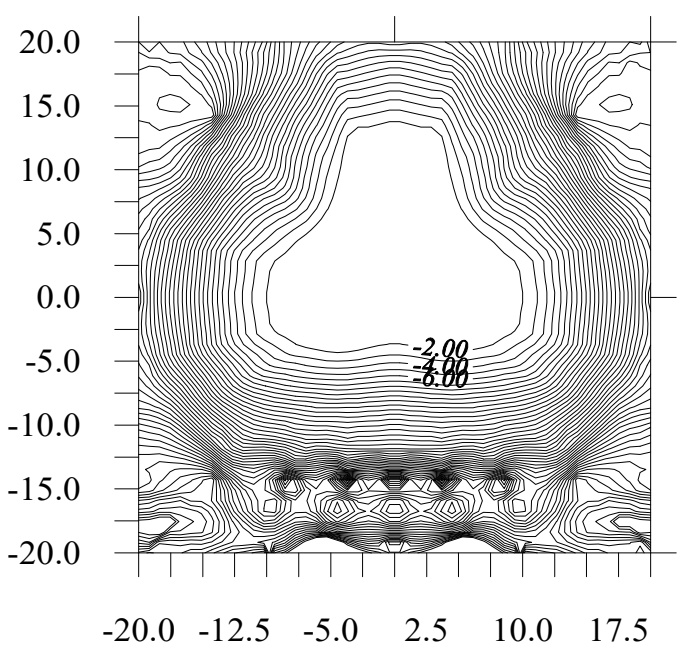

$b$

Fig. 5.4. Synthesis of contour DP

\section{Conclusions}

Note the main features and problems arising at investigations of othe class of problems reviewed in the article:

- The investigation of nonuniqueness and branching of existing solutions, which depend on the physical parameters of the problem is the main difficulty at solving this class of problems. As follows from the researches presented, in particular, for a special case in $[7,13$, 16], when $F\left(s_{1}, s_{2}\right)=F_{1}\left(s_{1}\right) \cdot F_{2}\left(s_{2}\right)$, the quality of existing solutions increases significantly with growth of parameters $c_{1}, c_{2}$. However, to obtain the best approximation to the given amplitude DP $F\left(s_{1}, s_{2}\right)$ at relatively small values of the parameters $c_{1}, c_{2}$ describing the sizes of aperture, allowing to confine by investigations of the first few branching points (lines), is essential in the synthesis problems of radiating systems.

- At finding the solutions of (3.9) by the successive approximations method in the case of the even by both arguments (or one argument) functions $F\left(s_{1}, s_{2}\right)$ to obtain solution of certain 
type it is necessary to choose an initial approximation, which belongs to the corresponding invariant set of nonlinear operators $B_{1}$ and $B_{2}$.

- On the base of computational experiments it is revealed that the branching-off complex solutions, which exist at small sizes of aperture, increase the efficiency of synthesis within $20-40 \%$ compared with the real (primary) solutions. The presence of different by structure but identical by efficiency solutions (in the sense of value of the corresponding functional), provides for practice the possibility of choosing one of them that has a simpler physical realization. In addition, the branching-off solutions at conservation of the same efficiency which corresponds to real solutions allow to reduce the linear size of the aperture in the limits of 10 to 20 percent.

- The proposed numerical method of solution of nonlinear two-parameter spectral problems arising at investigation of nonlinear integral equations can be successfully applied, in particular, to solving the linear and nonlinear two-parameter spectral problems concerning matrix equations and ordinary differential equations of the second $n$-th order with nonlinear occurrence of the spectral parameters into coefficients of equations and boundary conditions.

- A mathematical analogy between the synthesis problems of acoustic and electromagnetic antennas and synthesis problems of radio allows to use developed methods and numerical algorithms in the above sections of acoustics, radio physics and radio engineering.

\section{References}

[1] G. T. Markov, B. M. Petrov and G. P. Grudinskaja, Electrodynamics and Propagation of Radio Waves, Sov. Radio, Moscow, 1979.

[2] G. T. Markov and A. F. Chaplin, Excitation of Electromagnetic Waves, Radio and communications, Moscow, 1983.

[3] V. B. Zhukov, Calculation of the Hydroacoustic Antennas according to the Directivity Pattern, Sudostroienije, Leningrad, 1977.

[4] A. F. Chaplin, Analysis and Synthesis of Antenna Array, Vyshchaya shkola, Lvov, 1987.

[5] G. T. Markov and D. N. Sazonov, Antennas, Energiya, Moscow, 1975.

[6] E. G. Zelkin and V. G. Sokolov, Synthesis Methods of Antenna Arrays and Antenna with continuous aperture, Sov. Radio, Moscow, 1980. 
[7] P. O. Savenko, Nonlinear Problems of Radiating Systems Synthesis (Theory and Methods of Solution), IAPMM of NASU, Lviv, 2002.

[8] M. Minkovich and V. P. Yakovlev, Theory of Synthesis of Antennas, Sov. Radio, Moscow, 1969.

[9] B. Z. Katsenelenbaum, "Approximability Problem of the Electromagnetic Field", Uspekhi phys. nauk, vol. 164, no. 9, pp. 983-993, 1994.

[10] G. V. Aleksejev, "About Incorrect of the First Kind Nonlinear Operator Equation of Antennas Synthesis Theory", J. of Comp. Math. and Math. Phys., vol. 19, no. 6, pp. $1590-1595$.

[11] T N. Tikhonov and V. Y. Arsenin, The Methods of Solution of Incorrect Problems, Nauka, Moscow, 1979.

[12] V. K. Ivanov, V. V. Vasin and V. P. Таnana Иванов, The Theory of Linear Incorret Problems and its Applications, Nauka, Moscow, 1978.

[13] M. I. Andriychuk, N. N. Voitovich, P. A. Savenko and V. P. Tkachuk, Antenna Synthesis According to Amplitude Directivity Pattern: Numerical Methods and Algorithms, Naukova Dumka, Kiev, 1993.

[14] V. A. Trenogin, Functional Analysis, Nauka, Moscow, 1980.

[15] N. N. Voitovich and P. A. Savenko, "Generalized Criterion of Proximity Diagram in the Antennas Synthesis Problem by Method V. V. Semjonova", Radiotekhn. and electron., vol. 17, no. 9, pp. 1794-1798, 1973.

[16] N. N. Voitovich and P. A. Savenko, "Branching of Solutions of the Antenna Synthesis Problem According to the Prescribed Amplitude Directivity Pattern", Radiotekhn. and electron., vol. 21, no. 4, pp. 723-729, 1976.

[17] N. N. Voitovich and P. A. Savenko, "Synthesis of Antennas According to the Prescribed Amplitude Directivity Pattern and Associated Problems of Quasi-optics (review)", Radiotekhn. and electron, vol. 24, no. 8, pp. 1465-1500, 1979.

[18] P. A. Savenko, "Numerical Solution of One Class of Nonlinear Problems of Synthesis Theory of Radiating Systems”, J. of Comp. Math. and Math. Phys., vol. 40, no. 6, pp. 929-939, 2000.

[19] P. O. Savenko, "About the Existence of Solutions of One Class of Nonlinear Inverse Problems of Mathematical Physics Concerning Synthesis of Radiating Systems", Doklady Akad. Nauk of Ukraine, no. 8, pp. 48-52, 1994. 
[20] N. Kolmogorov and S. V. Fomin, Elements of the Theory of Functions and Functional Analysis, Nauka, Moscow, 1968.

[21] M. M. Vainberg, "Variational Method and Method of Monotone Operators in the Theory of Nonlinear Equations", Nauka, Moscow, 1972.

[22] P. A. Savenko, "Branching of Solutions of the Antenna Synthesis Problem According to the Prescribed Amplitude Directivity Pattern with Use of Regularizing Functionals", Izv. Vysch. uch. zaved. Radioelectronica, vol. 39, no. 2, pp. 35-50, 1996.

[23] F. Verlan and V. S. Sizikov, Integral Equations: methods, algorithms, programs. Reference textbook, Naukova Dumka, Kyiv, 1986.

[24] P. O. Savenko, "Synthesis of Linear Antenna and Antenna Arrays with Use of Regularizing Functionals", Proc. of Int. Seminar/Workshop "Direct and Inverse Problems of Electromagnetic and Acoustic Wave Theory", IAPMM of NASU, Lviv, pp. 62-63, 1995.

[25] P. O. Savenko. and V. J. Anokhin, "Numerical Optimization of Shape of Flat Aperture and Field According to the Prescribed Amplitude Directivity Pattern", Teoret. Electrotekh., Lviv, LDU, vyp. 53, pp. 118-126, 1996.

[26] P. O. Savenko and V. J. Anokhin, "Synthesis of the Surface Antenna for a Given Power Pattern", VI-th Int. Conf. "Mathematical Methods in Electromagn. Theory", Lviv, Ukraine, Sept. 10-13, 1996, Lviv, pp. 525-528, 1996.

[27] V. I Dmitriev and N. I. Berezina, Numerical Methods for Solving the synthesis of Radiating Systems, Izd. MGU, Moscow, 1986.

[28] P. A. Savenko, "Synthesis of Linear Antenna Arrays According to the Prescribed Amplitude Directivity Pattern”, Izv. Vysch. Uch. Zaved., Radiophys., vol. 22, no. 12, pp. $1498-1504,1979$.

[29] P. A. Savenko and L. M. Pasnak, "Numerically-analytical Method of Synthesis of Linear Antenna Arrays of Vibrators According to the Prescribed Amplitude Directivity Pattern", Izv. Vysch. Uch. Zaved., Radiophys., vol. 40, no. 12, pp. 11-25, 1997.

[30] P. A. Savenko and L. M. Pasnak, "The Numerical Solution of Nonlinear Synthesis Problem of Antenna Array of Curved Radiator with Account of their Mutual Influence", Izv. Vysch. Uch. Zaved., Radioelectr., vol. 43, no. 8, pp. 1-15, 2000. 
[31] P. A. Savenko and M. D. Tkach, "Synthesis of Flat Microstrip Antenna Array According to the Prescribed Energy Directivity Pattern, Izv. Vysch. Uch. Zaved., Radioelectr., vol. 44, no. 6, pp. 19-32, 2001.

[32] L. M. Pasnak and P. O. Savenko, "Nonlinear Synthesis Problems of Antenna Arrays Considering Mutual Influence of Scatterers”, Millennium Conference on Antennas \& Propagation, Vol. 1. Davos, Switzerland, 9-14 April 2000, Davos, p. 201, 2000.

[33] L. M. Pasnak and P. O. Savenko , "Synthesis of Antenna Arrays with Regard to the Radiators Mutual Coupling and Vector Characteristics of Electromagnetic Fields", III Int. Conf. on Antenna Theory and Techniques (ICATT'99). Sevastopol, September 8-11, 1999, Sevastopol, pp. 135-137, 1999.

[34] L. M. Pasnak and P. O. Savenko, "Numerical Solution of Phase Synthesis Problem of Linear Array of Vibrators with Account their Mutual Influence”, Izv. Vysch. Uch. Zaved., Radioelectr.,vol. 40, no. 9, pp. 50-62, 1997.

[35] V.F.Kravchenko, L. P. Protsakh, P. A. Savenko and M. D. Tkach, "The Mathematical Features of the Synthesis of Plane Equidistant Antenna Arrays According to the Prescribed Amplitude Directivity Pattern”, Antennas, no. 3 (154), pp. 34- 48, 2010.

[36] P. A. Savenko and M. D. Tkach, "The Numerical Solution of the Nonlinear Synthesis Problem of Microstrip Antenna Array with Account the Mutual Influence of Radiators", Radiotekhn. And Electron., vol. 46, no. 1, pp. 58-65, 2001.

[37] P. O. Savenko, "Structure of Solutions of Synthesis Problem of Microstrip Array According to the Prescribed Energy Directivity Pattern", Matematychni Metody ta Fizyko-Mekhanichni Polya, vol. 46, no. 3, pp. 151-163, 2003.

[38] L. D. Bakhakh and S. D. Kremenetskii, Synthesis of Radiating Systems (theory and methods of calculations). Sov. Radio, Moscow, 1974.

[39] V. I. Dmitriev, A. S. Il'inskii and A. G. Sveshnikov, "Development of Mathematical Methods for the Study of Direct and Inverse Problems of Electrodynamics", Uspekhi math. Nauk, vol. 31, no. 6, pp. 123-141, 1976.

[40] V. I. Dmitriev and P. P. Sereda, "Mathematical Models and Method of Integral Equations in the Theory of Spiral Wire Antennas", Chislen. metody electodinam., Moscow, MGU, vyp. 4, pp. 55-65, 1980.

[41] A. S. Il'inskii, "Electrodynamic Theory of Antenna Arrays", Izv. Vysch. Uch. Zaved., Radioelectr., vol. 21, no. 2, pp. 9-21, 1978. 
[42] A. S. Il'inskii and I. V. Berezhnaja, "Investigation of the Current Distribution in the System Arbitrarily Located Vibrators", Vychislit. Metody and Program., Moscow, MGU, vyp. 20, pp. 263-268, 1973.

[43] L. M. Pasnak and P. O. Savenko , "Synthesis of Linear Equidistant Antenna Array of Interacting Vibrators According to the Prescribed Amplitude Directivity Pattern", Radiotekhn. fnd Electron., vol. 42, no. 5, pp. 567-572, 1997.

[44] P. O. Savenko and L. M. Pasnak, "Synthesis of Linear Antenna Array for According to the Prescribed Amplitude DP with Account of their Mutual Influence”, Visnyk Lvivs. Univ., vyp. 42, pp. 69-74, 1995.

[45] P. A. Savenko and L. M. Pasnak, "Numerical Solution of the Nonlinear Synthesis Problem of Antenna Array of Curved Radiators with Account their Mutual influence", Izv. Vysch. Uch. Zaved., Radioelectr., vol. 43, no. 8, pp. 1-15, 2000.

[46] P. O. Savenko "About one Method of the Synthesis Problems of Radiating Systems under the Given Power Directivity Pattern", Int. Conf. "Mathemat. Methods in Electromagn. Theory", Kharkiv, Ukraine, September 11-15, 2000, Kharkiv, pp. 618-620, 2000.

[47] P. A. Savenko, "About Structure of the Solutions of the Synthesis of Linear Antenna under the Given Energy Directivity Pattern”, Radiophys. and Radioastronomiya, vol. 5, no. 4,pp. 405-415, 2000.

[48] P. O. Savenko, "Numerical Solution of Synthesis Problems of Radiating Systems According to the Prescribed Power Directivity Pattern”, Proc. of 6th Int. Sem. "Direct and Inverse Problem of Electromagnetic and Acoustic Wave Theory", IAPMM of NASU, Lviv, pp. 141-145, 2001.

[49] P. A. Savenko, The Numerical Solution of the Inverse Synthesis Problems of Radiating Systems According to the Prescribed Power Directivity Pattern", J. of Comp. Math. and Math. Phys., vol. 42, no. 10, pp. 1495-1509, 2002.

[50] M. M. Vainberg, Variational Methods of Investigation of Nonlinear Operators, Gos. Izd. Tekhn.-teoret. Liter., Moscow, 1956.

[51] M. M. Vainberg, Functional Analysis, Prosveschenije, Moscow, 1979.

[52] P. O. Savenko, "Branching of solutions of the synthesis problem of linear antenna according to the prescribed amplitude directivity pattern in the space of Helder", Matematychni Metody ta Fizyko-Mekhanichni Polya, vol. 41, no. 2, pp. 35-44, 1998. 
[53] M. M. Vainberg and V. A. Trenogin, Theory of branching of solutions to nonlinear equations, Nauka, Moscow, 1969.

[54] N. N. Voitovich and P. A. Savenko, "About one integral equation of antenna synthesis theory", Matematychni Metody ta Fizyko-Mekhanichni Polya, vyp. 2, pp. 161-163, 1975.

[55] P. A. Savenko, "About the branching of the solutions of the synthesis problems of radiating systems with flat aperture according to the prescribed amplitude directivity pattern”, Izv. Vysch. Uch. Zaved., Radiophys, vol. 29, no. 3, pp. 339-345, 1986.

[56] P. O. Savenko, L. P. Protsakh and M. D. Tkach, "About the mean-square approximation of real finite non-negative continuous function with respect to two variables by module of double Fourier integral". I, Matematychni Metody ta Fizyko-Mekhanichni Polya, vol. 51, no. 1, pp. 53-64, 2008.

[57] P. O. Savenko, L. P. Protsakh and M. D. Tkach, "About the mean-square approximation of real finite non-negative continuous function with respect to two variables by module of double Fourier integral”. II // Matematychni Metody ta Fizyko-Mekhanichni Polya, vol. 51, no. 4, pp. 73-78, 2008.

[58] L. P. Protsakh , P. O. Savenko and M. D. Tkach, "Branching of solutions of the problem of mean-square approximation of real finite function with respect to two variables by modulus of double Fourier transformation", Matematychni Metody ta FizykoMekhanichni Polya, vol. 53, no. 3, pp. 3-7, 2010.

[59] I. I. Liashko, V. F. Yemelianov and A. K. Boyarchuk, Bases of classical and modern mathematical analysis, Vyshcha shkola, Kyiv, 1988.

[60] I. I. Privalov, Introduction to the theory of functions of complex variables, Nauka, Moscow, 1984.

[61] M. A. Krasnosel'skyy, G. M. Vainikko and P. P. Zabreiko, Approximate solution of operational equations, Nauka, Moscow, 1969.

[62] A. A. Abramov, L. F. Yukhno,” About a general nonlinear self-adjoint spectral problem for ordinary differential equations systems", J. of Comput. Math. And Math. Phys., vol. 49, no. 4, pp. 624-627, 2009.

[63] S. I. Solov'yev, “The Finite Element Method for Symmetric Eigenvalue Problems with Nonlinear Occurrence of Spectral Parameter”, J. of Comput. Math. And Math. Phys., vol. 37, no. 11, pp. 1311-1318, 1997. 
[64] L. P. Protsakh , P. O. Savenko , M. D. Tkach , "Numerical solving of two-dimensional nonlinear spectral problem arising in antenna synthesis with flat radiating aperture", Proc. of Xth Int. Seminar/Workshop "Direct and inverse problems of electromagnetic and acoustic wave theory". Lviv, September 23-25, 2005. Lviv, pp. 199-203, 2005.

[65] P. A. Savenko, L. P. Protsakh, "Implicit function method in solving the two-dimensional nonlinear spectral problem", Izv. Vysch. Uch. Zaved., Mathematics, no. 3 (546), pp. 4144, 2007.

[66] L. P. Protsakh, P. O. Savenko, "Implicit functions methods in solving the two-parameter linear spectral problems", Matematychni Metody ta Fizyko-Mekhanichni Polya, vol. 52, no. 2, pp. 42-49, 2009.

[67] P. O. Savenko, L. P. Protsakh, "Numerical solution of two-point boundary problem with two-dimensional nonlinear spectral parameter", Matematychni Metody ta FizykoMekhanichni Polya, vol.1, n0. 1, pp. 48-56, 2011.

[68] G. M. Vainikko, Analysis of discretized methods, Tartus. Gos. Universitet, Tartu, 1976.

[69] L. V. Kantorovich and G. P. Akilov, Functional analysis, Nauka, Moscow, 1977.

[70] V. I. Smirnov, Course of high mathematics, Vol. 2, Nauka, Moscow, 1974.

[71] P. O. Savenko, "The numerical algorithm of solving the generalized eigenvalues problem for completely continuous self-adjoint operators with nonlinear spectral parameter", Matematychni Metody ta Fizyko-Mekhanichni Polya, vol. 40, no. 1, pp. 146-150, 1997.

[72] P. O. Savenko, L. P. Protsakh, "Variational approach to solving the nonlinear vector spectral problem for the case of self-adjoint positive semi-definite operators", Dopovidi of NASU, no.6, pp. 26-31, 2004.

[73] P. A. Savenko, "The branching of the solutions of the synthesis problems of radiating systems with flat aperture according to the prescribed amplitude directivity pattern", Izv. Vysch. Uch. Zaved., Radiophys, vol. 29, no. 3, pp. 339-345, 1986.

[74] L. P.Protsakh, P. O. Savenko, Tkach M. D. "Branching of solutions for the problem of mean square approximation of a real finite function of two variables by the modulus of double Fourier transformation", Journal of Mathematical Sciences, Vol. 180, No. 1, pp. 51-67, 2012.

[75] P. A. Savenko, "The numerical solution of one class of nonlinear synthesis problems of radiating systems", // J. of Comput. Math. And Math. Phys., vol. 40, no. 6, pp. 929-939, 2000 . 
[76] P. O. Savenko, "About Convergence of the Iterative Process in a Nonlinear Synthesis Problems of Radiating Systems under the Use Smoothing Functionals", Matematychni Metody ta Fizyko-Mekhanichni Polya, vol. 43, no. 2, pp. 105-111, 2000.

[77] P. O. Savenko, V. J. Anokhin, "Synthesis of Amplitude-Phase Distribution and Shape of a Plane Antenna Aperture for a Given Power Pattern", IEEE Trans. on Antennas and Propag, vol. 45, no. 4, pp. 744-747, 1997.

[78] V. J. Anokhin , P. O. Savenko, "Synthesis of the Contour Directivity Pattern Without Critical Zones", URSI Int. Symp. Electromagn. Theory, St. Peterburg, Russia, May 2326, 1995, St. Peterburg., pp. 197-199, 1995.

[79] P. O. Savenko, V. J. Anokhin, "Synthesis of the Surface Antenna for a Given Power Pattern", 6th Int. Conf. "Mathematical Methods in Electromagn. Theory", Lviv, Ukraine, Sept. 10-13, 1996, Lviv, pp. 525-528, 1996.

[80] B. Podlevskyi, P. Savenko, "Synthesis of the Multibeams Reflector Antenna Directivity Patterns on the Given Magnitude", 6th Int. Conf. "Mathematical Methods in Electromagn. Theory", Lviv, Ukraine, Sept. 10-13, 1996, Lviv, pp. 521-524, 1996.

[81] B. Podlevskyi, P. Savenko, "Synthesis of the Variable Shape Contoured Directivity Patterns of the Hybrid Reflector Antennas", Trans Black Sea Region Symposium on Applied Electromagnetism (April 17-19, 1996, Metsovo, Greece)., N.T.U.A. PRES, Athens., p. 20, 1996.

[82] B. Podlevskyi, P. Savenko, "Synthesis of the Variable Shape Contoured Directivity Patterns of the Hybrid Reflector Antennas", Electromagnetics, vol.18, no. 5, pp. 507527,1998 . 